\title{
CAMBIO DE METODOLOGÍA EN LA ENCUESTA DE CONFIANZA DE LOS CONSUMIDORES DE COSTA RICA
}

\section{CHANGE OF METHODOLOGY IN THE COSTA RICA CONSUMER CONFIDENCE SURVEY}

\author{
Johnny Madrigal-Pana ${ }^{1}$ \\ Sebastián Flores-Alvarado ${ }^{2}$ \\ María Fernanda Benavides-Prado ${ }^{3}$ \\ Manuel Fiallos-Gonzáles ${ }^{4}$
}

\section{RESUMEN}

En mayo del 2019, se modificó la metodología de la Encuesta de Confianza de los Consumidores, cambiando de la telefonía fija a la móvil, por lo que las cuatro encuestas previas se realizaron con ambos métodos para investigar el efecto en el Índice de Confianza del Consumidor (ICC), entre otras estadísticas de interés. En tres de las encuestas no se encontró evidencia de diferencias en las magnitudes del ICC. Además, la disminución de la confianza que registró el índice de mayo a noviembre se dio con ambos tipos de encuesta (-16,8 puntos con la fija y $-16,0$ puntos con la móvil) y el aumento de la confianza en las encuestas siguientes fue similar (6,6 y 6,4, respectivamente). Se concluye que, usando ambas metodologías, la magnitud de las estimaciones del ICC, $y$ de otros indicadores de interés, no mostraron diferencias estadísticamente significativas en tres de las cuatro encuestas y que también reproducen la misma tendencia.

PALABRAS CLAVE: ESTADÍSTICA, CONFIANZA DEL CONSUMIDOR, CAMBIO METODOLÓGICO, ENCUESTA TELEFÓNICA, ENCUESTA CELULAR.

CLASIFICACIÓN JEL: C830

1 Universidad de Costa Rica, Escuela de Estadística; Código Postal: 11501-2060; San José, Costa Rica; johnny.madrigalpana@ucr. ac.cr

2 Universidad de Costa Rica, Escuela de Estadística; Código Postal: 11501-2060; San José, Costa Rica; sebastian.floresalvarado@ucr. ac.cr

3 Universidad de Costa Rica, Escuela de Estadística; Código Postal: 11501-2060; San José, Costa Rica; maria.benavidesprado@ucr ac.cr 


\begin{abstract}
In May 2019, the methodology of the Consumer Confidence Survey was modified, changing from fixed to mobile telephony, so the previous four surveys were conducted with both methods to investigate the effect on the Consumer Confidence Index (ICC), among other statistics of interest. In three of the surveys no evidence of differences in the magnitudes of the ICC was found. In addition, the decrease in confidence recorded by the index from May to November was with both types of survey (-16,8 points with the fixed and $-16,0$ points with the mobile) and the increase in confidence in the following surveys was similar (6,6 and 6,4, respectively). It is concluded that, in general, the magnitude of the ICC estimates, and other indicators of interest, is similar using both methodologies and also reproduces the same trend. KEY WORDS: STATISTICS, CONSUMER CONFIDENCE, METHODOLOGICAL CHANGE, TELEPHONE SURVEY, CELLPHONE SURVEY

JEL: C830
\end{abstract}

\title{
I. INTRODUCCION
}

En Costa Rica, la confianza de los consumidores es medida periódicamente, desde el año 2002, por la Escuela de Estadística de la Universidad de Costa Rica. La información que se deriva de esta encuesta es diversa e incluye percepciones y expectativas de la situación económica personal, del momento de compra de bienes duraderos (casa y vehículo personal), de la política económica y social, del tipo de cambio y precio de los combustibles, entre otros. El principal producto que aporta es el Índice de Confianza del Consumidor (ICC), estadística que constituye un "termómetro" trimestral de la percepción que tienen los consumidores de la situación económica, para establecer el grado de pesimismo u optimismo que experimentan.

El esquema de pensamiento para realizar la encuesta proviene del Survey Research Center de la Universidad de Michigan (U.S.A), donde se calcula el ICC desde mediados de 1945 (Curtin, 1982, p. 341). Para realizarla se establece que el optimismo hacia la economía genera confianza entre los consumidores y, como consecuencia, aumenta el deseo de realizar gastos y contraer deudas, y que, por el contrario, la incertidumbre produce pesimismo $y$, por ello, el deseo de reducir los gastos y reorganizar las reservas financieras. Como precursor de este pensamiento, George Katona, en su libro The Mass Consumption Society (Katona, 1964), cuyo pensamiento fue sintetizado por Richard Curtin en su artículo Indicators of Consumer Behavoir (Curtin, 1982, p. 341), postuló entonces que cuando muchos consumidores cambian al mismo tiempo de una perspectiva a otra, surge un cambio generalizado en su comportamiento de compra y esto afecta, positiva o negativamente, la actividad económica. Además, estableció que, debido a que el cambio en las actitudes y las expectativas de los consumidores ocurre previo al suceso, se considera que el ICC puede actuar como un indicador adelantado de la actividad económica. De esta manera, el planteamiento le asigna importancia al poder del consumidor, ya que puede influir en el comportamiento cíclico de la economía, en su tasa de crecimiento y en los períodos de inflación o de deflación. Teóricamente, esto lo hacen los consumidores a través de la demanda de bienes de consumo discrecional, la que está influenciada, $y$ algunas veces determinada, por su deseo de compra, esto es, por sus motivaciones, actitudes $y$ expectativas.

Después de varios años de realizarla, la Encuesta de Confianza de los Consumidores (ENCON) se convirtió en un insumo de interés para investigadores, académicos, entidades financieras públicas y privadas, políticos y medios de comunicación. Además, desde mediados del 2015 forma parte del Sistema Estadístico Nacional (SEN) y se adoptó como una estadística nacional que es reportada a la Organización para la Cooperación y el Desarrollo Económico (OCDE), como consta en el Plan Estadístico Nacional (INEC, 2018). 
Metodológicamente hablando, la ENCON se realiza, desde su inicio en setiembre del 2002, usando la técnica de entrevista telefónica para recolectar la información, por lo que para cada encuesta se selecciona una muestra de teléfonos residenciales o de telefonía fija ${ }^{5} y$, después de 16 años de ejecución, en febrero del 2019, se acumuló una serie de 61 encuestas, en las que aspectos metodológicos como el contenido del cuestionario, el tamaño de la muestra, la técnica de selección de la muestra y del informante, entre otros, permanecieron sin cambios garantizando la comparabilidad de la información.

Pese a disponer de una serie de datos comparable por un período importante de años, a inicios del año 2018, la Escuela de Estadística consideró impostergable revisar algunos aspectos metodológicos de la encuesta, para ajustarlos a la situación actual del país en materia de telefonía. Esta iniciativa surgió porque la tenencia de la telefonía fija en los hogares está disminuyendo sustancialmente, lo que obliga y hace inaplazable empezar a utilizar la móvil para desarrollar la encuesta.

Una de las interrogantes más importantes que surgió para la investigación fue si este cambio en la metodología alteraría de alguna manera la serie de datos del ICC y, si la respuesta fuera positiva, cómo lo haría y cuál sería el efecto en la colección de encuestas ya realizada. Es necesario aclarar que, desde un principio se planteó que, independientemente del impacto del cambio metodológico, la modificación se haría, pues el interés estuvo centrado en el mejoramiento de la medición de las estadísticas derivadas de la encuesta, particularmente del ICC.

La necesidad de revisar y mejorar los principales indicadores proporcionados por la ENCON surge precisamente por la pérdida de cobertura geográfica que está experimentando la encuesta al seguir usando la telefonía fija, como se comprobará más adelante, lo que podría estar produciendo estimaciones para un grupo particular de hogares. Si la población que actualmente cubre la encuesta (personas residentes en hogares con telefonía fija) posee valoraciones y expectativas en el campo económico diferentes a las manifestadas en todos los hogares de Costa Rica, en los que casi el 100\% posee al menos un celular, entonces esta situación debe corregirse con el cambio metodológico. Además, debe tomarse en cuenta que, si esas valoraciones y expectativas son similares entre ambos grupos, el cambio también tendrá que realizarse, pues se espera una disminución en la cobertura de hogares con telefonía fija.

Debido a lo anterior, el objetivo de esta investigación fue analizar en la ENCON el efecto que tendrá cambiar la telefonía fija por la móvil, para continuar brindando información oportuna y de calidad sobre el ámbito nacional a las personas y entidades que lo requieran. Para lograrlo, se explicará la metodología utilizada en la investigación, luego se hará una descripción de los resultados en cuatro apartados y se finalizará con las conclusiones. Es importante aclarar, para finalizar este apartado, se consideró esencial analizar el impacto que tendrá el cambio metodológico en el ICC, pues se decidió que a partir de mayo del 2019 la encuesta seguirá ejecutándose con el uso de la telefonía móvil.

5 Desde setiembre de 2002 (fecha de la primera encuesta), se ejecutaron seis encuestas semestrales (marzo y setiembre), aspecto que se modificó a partir de agosto del 2005, pues se empezó a realizar trimestralmente (febrero, mayo, agosto y noviembre). 


\section{METODOLOGÍA}

Para cuantificar los efectos en las estimaciones de la encuesta, prestando especial atención al ICC, se desarrolló el siguiente procedimiento:

1. En los meses de mayo, agosto y noviembre del 2018, así como en febrero del 2019, se continuó realizando la ENCON con la metodología ya establecida: usando telefonía fija y entrevistando al principal sostén económico del hogar.

2. En los mismos meses se ejecutó una encuesta paralela usando una muestra de telefonía móvil y considerando como informante a la persona que tiene 18 años o más que usa celular.

En síntesis, la Escuela de Estadística ejecutó en un año cuatro encuestas paralelas para comparar telefonía fija, cuyo informante es el principal sostén económico del hogar (método tradicional), con la telefonía móvil, cuyo informante es una persona de 18 años o más que usa celular (cambio metodológico). El por qué adoptar esta estrategia y estos cambios se explica a continuación.

Un primer punto de aclaración es el cambio del tipo de telefonía. Al respecto, el desarrollo tecnológico que está experimentando el planeta en diversos campos, particularmente en lo referente a las tecnologías de información y comunicación, hizo que en Costa Rica, a lo largo de los años, la telefonía móvil ganara más usuarios y la fija sea cada vez menos requerida. Esto es fácil demostrarlo, pues en el país, en el año 2002 (fecha de la primera ENCON), 62\% de los hogares poseía telefonía fija, cifra que disminuyó a 32\% en el 2018 (Gráfico 1). Por el contrario, mientras en el 2002 la telefonía móvil estaba solamente en el $27 \%$ de los hogares, para el 2018 aumentó a 99\%. Si bien es cierto, esta información hace referencia a los hogares, en el 2017 se estimó que 96\% de la población de 18 años y más usaba al menos un dispositivo de este tipo en Costa Rica (Escuela de Estadística, 2017). Este hecho, que surge como un evento positivo desde el punto de vista del aumento de las tecnologías de comunicación entre las personas, originó la necesidad de migrar de la telefonía fija a la móvil.

\section{GRÁFICO 1 \\ PORCENTAJE DE TENENCIA DE TELEFONÍA FIJA Y MÓVIL EN EL HOGAR DEL AÑO 2002 AL 2018}

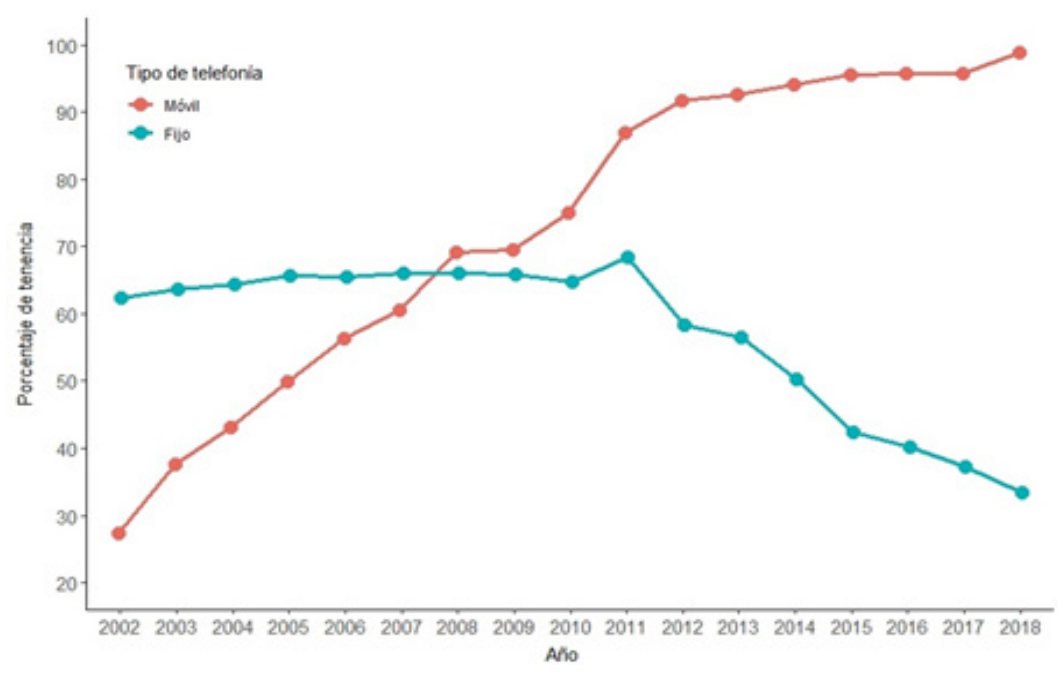

Fuente: : Elaboración a partir de los datos del Instituto Nacional de Estadística y Censos (2002) - (2009) e Instituto Nacional de Estadística y Censos (2010) - (2018). 
Un segundo aspecto que es necesario aclarar es lo relacionado al número de encuestas, pues se consideró insuficiente desarrollar una única medición paralela para comparar. Se tomó la decisión de ejecutar cuatro para contrastar las estimaciones con ambas metodologías en un año (cuatro trimestres), pues así se tendrá más confianza en las conclusiones referentes a la comparabilidad de las estimaciones, particularmente en lo relacionado con el ICC. Además, podrá verificarse si ambas metodologías proveen tendencias similares o diferentes de los indicadores, lo que no podría analizarse con una única medición.

Un tercer aspecto por aclarar es el procedimiento de selección de teléfonos. Para disponer de un marco muestral de teléfonos residenciales, desde el año 2002, el Instituto Costarricense de Electricidad (ICE) provee el listado de centrales telefónicas residenciales activas en todo el país y con esta información se construyen los denominados bancos telefónicos activos (primeros dígitos de los números telefónicos residenciales activos por central telefónica). Esta información permite estratificar los hogares del país en tres zonas: Gran Área Metropolitana (55\% de hogares), Resto del Valle Central (25\%) y Resto del País (20\%). Posteriormente, se selecciona una muestra aleatoria estratificada de 700 teléfonos fijos cada vez que se ejecuta la encuesta. Para hacerlo, se utiliza la técnica de Waksberg (Waksberg, 1978) que consiste en seleccionar números telefónicos al azar, utilizando un programa que escoge, en una primera etapa, los bancos telefónicos activos disponibles en el país (primeros cuatro dígitos de teléfonos activos) y en una segunda etapa, completa con números aleatorios los números telefónicos de la muestra. En la primera etapa se utilizan los primeros cuatro dígitos de los teléfonos activos para tratar de disminuir las llamadas improductivas que producen los teléfonos inexistentes o comerciales. En el caso de la telefonía móvil, se usa como marco muestral la información suministrada por la Superintendencia de Telecomunicaciones (SUTEL), de donde se obtienen los códigos activos de las diferentes empresas que brindan el servicio de telefonía móvil en Costa Rica (primeros dígitos). De esos códigos activos, se generan los bancos telefónicos $y$, posteriormente, se selecciona una muestra aleatoria de 700 teléfonos móviles en cada encuesta, usando también la técnica de Waksberg con el mismo fin que se hace para la telefonía fija. Esto hizo necesario construir un programa para generar los números telefónicos al azar. Se aclara que el procedimiento de la asignación de los números de telefonía móvil a los usuarios no permite estratificar la muestra geográficamente, como en el caso de la telefonía fija.

Un cuarto punto por aclarar es sobre el informante. En el caso de la telefonía fija, el informante es el principal sostén económico del hogar (miembro que aporta más recursos económicos para la manutención del hogar) o, en su defecto, y después de tres intentos, una persona adulta informada de la situación económica de la familia. No obstante, se consideró que al mantener esta práctica con la telefonía móvil podría aumentar el período de la recolección de los datos y, como consecuencia, fallar al compromiso de brindar los resultados de la encuesta el último miércoles del mes en que se ejecuta (meta de oportunidad).

Lo anterior sucedería porque el tiempo para localizar con telefonía móvil a una persona que sea principal sostén económico requeriría numerosas llamadas improductivas que deberán desecharse, pues una cantidad importante se hará a usuarios sin ese atributo. Esto sucederá porque responderán personas en edad escolar o colegial, jóvenes o adultos sin trabajo, amas de casa, hijos(as) que viven con sus padres, etc. En otras palabras, se sabe que quienes no poseen la característica de ser principal sostén económico también hacen uso del celular en magnitudes importantes, lo que aumentará las llamadas improductivas. Por ejemplo, en el año 2016 se encontró que 83\% de quienes tienen edad escolar y colegial (hombres y mujeres de 5 a 17 años) usaba celular (Instituto Nacional de Estadística y Censos, Ministerio de Cultura y Juventud, 2017, p. 89). Esta situación del número de llamadas improductivas para localizar al principal sostén económico en la telefonía móvil se evitó, cambiando el informante por una persona de 18 años o más que use celular al momento de la llamada, pues se consideró que todos son consumidores, con percepciones y expectativas en el campo económico y social. 
Por otra parte, desde el punto de vista de análisis, se presta particular importancia a los cambios que experimenta el ICC usando ambas metodologías, así como otros dos indicadores que se derivan de este: el Índice de Condiciones Económicas Actuales (ICEA) y el Índice de Expectativas Económicas (IEE). El cálculo del ICC se hace con las respuestas a cinco preguntas: dos referentes a las condiciones económicas actuales y otras tres relacionadas con las expectativas económicas (cuadro Anexo 1). Específicamente, las preguntas son:

1. ¿Podría decirme si, económicamente, usted y su familia están mejor o peor que hace un año?

2. Ahora, hablando sobre los artículos grandes que las familias compran para su hogar, tales como muebles, refrigeradoras, cocinas y televisores, entre otros. ¿Diría usted que, en términos generales, actualmente es un buen momento o un mal momento para que las familias compren estos artículos?

3. Y dentro de un año ¿considera que usted y su familia estarán, económicamente, mejor, peor o igual que ahora?

4. Y durante los próximos 12 meses ¿piensa usted que la situación económica de las empresas o negocios del país será mejor, peor o igual que ahora?

5. Pensando en general sobre el futuro ¿diría usted que en los próximos 5 años el país tendrá buenos tiempos, períodos de crisis o qué?

El ICC se escala de cero a 100, lo que se logra poniendo 100 a las respuestas favorables, 50 a las neutras o sin respuesta y 0 a las desfavorables, $y$ calculando finalmente un promedio simple con los cinco puntajes, cuya fórmula es: ICC $=\left(X_{1}+X_{2}+X_{3}+X_{4}+X_{5}\right) / 5$. El ICC tiene un rango de 0 a 100 , siendo el valor mínimo en cero, lo cual es el pesimismo más acentuado y su contraparte, el 100, es el optimismo mayor; por otra parte, la posición intermedia, cuando el valor está tendiendo a 50, se clasifica como la posición indecisa, neutral o con incertidumbre (Madrigal, 2012, p. 11).

Además, para el ICEA se consideran las preguntas que valoran la situación actual para la compra de bienes duraderos y cuál es la percepción de la familia en el ámbito económico respecto a hace un año (primeras dos preguntas) y hace que este índice brinde una valoración de la confianza que actualmente experimentan los consumidores en el ámbito económico; y para calcularlo se realiza también un promedio simple de las variables recodificadas, siendo su fórmula: $\operatorname{ICEA}=\left(X_{1}+X_{2}\right) / 2$. Además, para el IEE se toman las preguntas relacionadas con la situación económica de cada familia y de las empresas dentro de un año y de su visión de la situación económica del país dentro de los cinco años próximos (preguntas tres, cuatro y cinco), con lo cual se da una valoración sobre el futuro económico del país, por parte de los consumidores; la fórmula para el este índice es a partir de la variables recodificadas y también con un promedio simple y se expresa de la siguiente manera: IEE $=\left(X_{3}+X_{4}+X_{5}\right) / 3$. (Madrigal, 2012, p. 11).

Para establecer si las estimaciones presentan diferencias significativas entre encuestas paralelas del mismo mes, se calculan los intervalos de confianza ${ }^{6}$ ( $5 \%$ de significancia), usando en cada caso el diseño muestral correspondiente. Específicamente, en la encuesta con telefonía fija los errores estándar son calculados usando un diseño muestral complejo de conglomerados desiguales (bancos telefónicos) estratificados por zona. En el caso de la encuesta en telefonía móvil, al no haber estratos estadísticos, los errores estándar se calculan usando solamente un diseño complejo de conglomerados desiguales (bancos telefónicos).

Otros aspectos técnicos que deben aclararse al aplicar ambas metodologías son el uso de ponderadores, la duración de la entrevista y la ejecución del trabajo de campo. En lo referente al uso

6 Se aclara que el uso de intervalos de confianza para determinar diferencias significativas está basado en Gardner \& Altman (1986) y Foody (2009). 
de los ponderadores, cuando la entrevista se hace al principal sostén económico del hogar (telefonía fija), no se ejecutan ajustes o se construyen ponderadores, pues no se dispone de información para hacerlo. No obstante, con el cambio metodológico, al entrevistar personas de 18 años y más que usan celular, se ajusta la muestra final por no respuesta, utilizando la estructura por sexo, edad y escolaridad que registra anualmente la Encuesta Nacional de Hogares (ENAHO) del Instituto Nacional de Estadística y Censos (INEC).

La duración de la entrevista resultó prácticamente igual en ambos tipos de encuesta. Mientras que en la realizada con telefonía fija tuvo una duración media de 10 minutos y medio, en la móvil fue poco más de 11 minutos. Esta diferencia, que no es relevante para efectos de la recolección de la información, se debe a que, aunque ambos cuestionarios son prácticamente iguales, el de telefonía móvil contiene preguntas adicionales sobre características de la persona entrevistada, así como la provincia, cantón y distrito de residencia, información que es de utilidad para analizar la dispersión de la muestra de 700 entrevistas a lo largo y ancho de Costa Rica.

Finalmente, se capacitó a los entrevistadores para realizar las entrevistas, se explicaron los procedimientos de selección de números telefónicos y de registro de la información recolectada, entre otros. Para recolectar los datos, se usa un laboratorio equipado especialmente para este tipo de encuestas, con 16 computadoras en red, utilizando un sistema de entrevistas telefónicas asistido por computadora (computer-assisted telephone interviewing). Se hizo un énfasis particular en la selección del informante según el tipo de telefonía usada. En el trabajo de campo, aunque no se poseen datos al respecto, quienes realizaron las entrevistas comentaron que resultó más rápido concretar una entrevista con la telefonía móvil que con la fija. En otras palabras, entrevistar al principal sostén económico del hogar usando telefonía fija requiere más esfuerzo (no responden al teléfono, no está en la vivienda o debe llamar más tarde) que el realizado para entrevistar a una persona de 18 años y más que usa celular, pues al ser el usuario del teléfono lo que puede suceder es que, cuando responde, deje la entrevista pendiente o la rechace.

\section{RESULTADOS}

La comparación entre ambos tipos de encuesta, y entre las de un mismo tipo, se hará en cuatro apartados. El primero es para conocer las características sociodemográficas del informante y las socioeconómicas del hogar, entre ambos tipos de encuesta. Esto es importante para determinar los atributos de los informantes y de los hogares cubiertos con ambas metodologías. En el segundo apartado se comparan, entre ambas encuestas, los resultados del ICC y sus diferenciales, usando las características previamente mencionadas. La tercera compara entre ambos métodos la clasificación de los consumidores (optimistas, ambivalentes y pesimistas), así como del ICEA y el IEE. Un apartado final se ocupa de la representatividad de la muestra de 700 personas de 18 años y más que usa telefonía móvil, lo que es de interés, pues será la metodología que seguirá utilizándose.

\section{Características del informante y del hogar}

Cuando la encuesta se hizo con telefonía fija, el porcentaje de hombres que son principal sostén económico del hogar varía entre 44\% y 50\% en las cuatro encuestas (cuadro 1). En cambio, en la telefonía móvil, los hombres de 18 años y más que usan celular son, en promedio, 48\% y las magnitudes varían poco entre encuestas. Esto muestra que en ambos tipos de encuesta los hombres responden el cuestionario en poco menos del $50 \%$ de las entrevistas, aunque el porcentaje es más variable entre encuestas de telefonía fija que en la móvil. Este último aspecto puede corroborarse observando las diferencias porcentuales entre hombres y mujeres para cada encuesta. 
Algo distinto sucede con la edad, pues las distribuciones porcentuales son bastante diferentes entre ambos tipos de encuesta y casi no varían entre meses de un mismo tipo de encuesta. Por ejemplo, mientras que con la telefonía fija poco más del $65 \%$ de los informantes tiene 50 años o más de edad, en la móvil es cercana al 35\%. Esto indica que la encuesta con telefonía fija concentra personas de mayor edad (principal sostén económico) en mayor proporción que la hecha con telefonía móvil (personas de 18 años o más que usa celular). Esto también se observa en las diferencias porcentuales entre el grupo de edad mayor y el menor. Además, las diferencias por educación también son evidentes entre ambos tipos de encuesta y bastante parecidas entre las de un mismo tipo. Esto se afirma porque al usar telefonía fija la magnitud que posee educación universitaria ronda 44\%, mientras que en la de telefonía móvil es aproximadamente $22 \%$.

\section{CUADRO 1 \\ DISTRIBUCIONES PORCENTUALES DE LAS CARACTERÍSTICAS SOCIODEMOGRÁFICAS DEL INFORMANTE POR TIPO DE TELEFONÍA SEGÚN MES DE ENCUESTA}

\begin{tabular}{lccccccccc}
\hline \multirow{2}{*}{$\begin{array}{l}\text { Características } \\
\text { sociodemográficas }\end{array}$} & \multicolumn{2}{c}{ Mayo 2018 } & \multicolumn{2}{c}{ Agosto 2018} & \multicolumn{2}{c}{ Noviembre 2018} & \multicolumn{2}{c}{ Febrero 2019 } \\
\cline { 2 - 9 } Total & \multicolumn{1}{c}{ Fija } & Móvil & Fija & Móvil & Fija & Móvil & Fija & Móvil \\
Sexo & $\mathbf{1 0 0 , 0}$ & $\mathbf{1 0 0 , 0}$ & $\mathbf{1 0 0 , 0}$ & $\mathbf{1 0 0 , 0}$ & $\mathbf{1 0 0 , 0}$ & $\mathbf{1 0 0 , 0}$ & $\mathbf{1 0 0 , 0}$ & $\mathbf{1 0 0 , 0}$ \\
Hombre & & & & & & & & \\
Mujer & 50,4 & 47,9 & 43,8 & 47,8 & 49,2 & 47,9 & 44,0 & 47,6 \\
Diferencia \%H-\%M & 49,6 & 52,1 & 56,2 & 52,2 & 50,8 & 52,1 & 56,0 & 52,4 \\
Edad & 0,8 & $-4,2$ & 12,4 & $-4,4$ & $-1,6$ & $-4,2$ & $-12,0$ & $-4,8$ \\
18 a 34 & & & & & & & & \\
34 a 50 & 7,9 & 37,9 & 8,3 & 37,9 & 8,3 & 37,9 & 9,5 & 37,1 \\
50 y más & 26,7 & 26,4 & 25,3 & 26,5 & 23,7 & 26,4 & 23,1 & 26,5 \\
Diferencia \%50-\%18 & 65,4 & 35,7 & 66,4 & 35,6 & 68,0 & 35,7 & 67,5 & 36,4 \\
Educación & 57,5 & $-2,2$ & 58,1 & $-2,3$ & 59,7 & $-2,2$ & 58,0 & $-0,7$ \\
Primaria & & & & & & & & \\
Secundaria & 30,6 & 39,9 & 34,1 & 39,9 & 30,1 & 39,9 & 30,4 & 39,3 \\
Universitaria & 25,1 & 37,5 & 22,1 & 37,4 & 22,7 & 37,5 & 27,1 & 37,6 \\
Diferencia \%U-\%P & 44,3 & 22,6 & 43,8 & 22,7 & 47,2 & 22,6 & 42,4 & 23,1 \\
\hline
\end{tabular}

Fuente: Elaboración propia con base en los datos de la ENAHO 
Adicional a las características sociodemográficas del informante, también se dispone de algunas características socioeconómicas de los hogares (cuadro 2). La capacidad de gasto discrecional en el hogar ${ }^{7}$, en la encuesta con telefonía fija, varía entre 50\% y 58\% (en las cuatro mediciones) y en el caso de la encuesta con telefonía móvil varía entre 30\% y 38\%. Algo similar sucede con el ingreso total mensual que reciben los miembros del hogar, pues en las encuestas con telefonía fija cerca de la mitad afirma tener un ingreso mensual de 500 mil o más y esa cifra ronda 34\% en las hechas con telefonía móvil. El ingreso subjetivo ${ }^{8}$ también muestra una tendencia similar, pues con la telefonía fija se obtiene que el porcentaje de hogares que puede ahorrar con el ingreso mensual que recibe (25\% en promedio de las cuatro encuestas) supera al obtenido con la telefonía móvil (19\% en promedio). Es decir, socioeconómicamente hablando, la telefonía fija provee indicadores de ingreso más favorables que los obtenidos con la telefonía móvil.

\section{CUADRO 2 \\ DISTRIBUCIONES PORCENTUALES DE LAS CARACTERÍSTICAS SOCIOECONÓMICAS DEL HOGAR POR TIPO DE TELEFONÍA SEGÚN MES DE ENCUESTA}

\begin{tabular}{|c|c|c|c|c|c|c|c|c|}
\hline \multirow{2}{*}{$\begin{array}{l}\text { Características } \\
\text { socioeconómicas }\end{array}$} & \multicolumn{2}{|c|}{ Mayo 2018} & \multicolumn{2}{|c|}{ Agosto 2018} & \multicolumn{2}{|c|}{ Noviembre 2018} & \multicolumn{2}{|c|}{ Febrero 2019} \\
\hline & Fija & Móvil & Fija & Móvil & Fija & Móvil & Fija & Móvil \\
\hline Total & 100 & 100 & 100 & 100 & 100 & 100 & 100 & 100 \\
\hline \multicolumn{9}{|l|}{ Gasto discrecional $^{1}$} \\
\hline No & 50,0 & 61,9 & 58,2 & 63,1 & 55,8 & 69,1 & 54,3 & 66,3 \\
\hline $\mathrm{Si}$ & 50,0 & 38,1 & 41,8 & 36,9 & 44,2 & 30,9 & 45,7 & 33,7 \\
\hline Diferencia \%S-\%N & 0,0 & $-23,8$ & $-16,4$ & $-26,2$ & $-11,6$ & $-38,2$ & $-8,6$ & $-32,6$ \\
\hline \multicolumn{9}{|l|}{ Ingreso mensual } \\
\hline Menos de 500.000 & 49,2 & 65,6 & 50,8 & 66,4 & 49,2 & 65,5 & 51,3 & 67,4 \\
\hline 500.000 o más & 50,8 & 34,4 & 49,2 & 33,6 & 50,8 & 34,5 & 48,7 & 32,6 \\
\hline Diferencia $\%<$ de- $\% 500 \mathrm{mil}+$ & 1,6 & $-31,2$ & $-1,6$ & $-32,8$ & 1,6 & $-31,0$ & $-2,6$ & $-34,8$ \\
\hline \multicolumn{9}{|l|}{ Ingreso subjetivo } \\
\hline Tienen grandes dificultades & 8,9 & 11,0 & 4,0 & 9,5 & 10,4 & 16,6 & 6,4 & 16,9 \\
\hline Tienen dificultades & 17,4 & 26,0 & 24,8 & 27,0 & 20,7 & 24,3 & 21,9 & 25,5 \\
\hline Sin grandes dificultades & 45,8 & 42,2 & 47,8 & 49,1 & 44,2 & 40,3 & 49,4 & 37,3 \\
\hline Pueden ahorrar & 27,9 & 20,8 & 23,5 & 14,4 & 24,7 & 18,8 & 22,3 & 20,3 \\
\hline Diferencia (\%Pa-\%Tgd) & 19,0 & 9,8 & 19,5 & 4,9 & 14,3 & 2,2 & 15,9 & 3,4 \\
\hline
\end{tabular}

1 Es un indicador de la capacidad que manifestó el informante de realizar, en el corto plazo, al crédito o al contado, compras de al menos uno de los siguientes bienes: artículos grandes para el hogar (juego de muebles, refrigeradora, cocina, televisor u otro similar), microcomputadora, terreno, viaje de placer, vehículo y casa.

Fuente: Elaboración propia a partir de los datos de la Escuela de Estadística, Universidad de Costa Rica (2019)

$7 \quad$ Hace referencia a la capacidad que tienen en el hogar de comprar, en el corto plazo, al crédito o al contado, artículos grandes para el hogar (juego de muebles, refrigeradora, cocina, televisor u otro similar), microcomputadora, terreno, viaje de placer, vehículo $y$ casa. Se trata de construir un proxy de la capacidad de gasto discrecional.

8 ¿Usted diría que el salario o ingreso total que su familia recibe mensualmente les alcanza o no les alcanza para vivir? 


\section{Resultados del ICC y diferenciales}

Una revisión del gráfico 2 muestra, en primera instancia, que las cuatro encuestas con telefonía fija parecieran producir estimaciones un poco más altas del ICC que las obtenidas con la móvil, aunque sus magnitudes son bastante cercanas entre ambos tipos de encuesta, como también puede observarse en el cuadro 3. Por este motivo, se calcularon los intervalos de confianza para cada par de encuestas paralelas y se corroboró que en tres no se posee evidencia de diferencias significativas (gráfico 2) y solo en agosto de 2018 las estimaciones del ICC resultaron estadísticamente diferentes (una diferencia de -4,4 puntos entre la estimación de la telefonía fija y la móvil, cuadro 3). En otras palabras, en tres de las cuatro encuestas paralelas realizadas, el ICC presentó magnitudes similares con ambas metodologías.

Un resultado relevante es que la tendencia del ICC en ambos tipos de encuesta es similar. Es decir, la disminución que experimentó el ICC de mayo a noviembre de 2018 se produjo en ambos tipos de encuesta (-16,8 puntos en la telefonía fija y -16,0 puntos en la móvil) y el aumento de la confianza registrado de noviembre del 2018 a febrero del 2019, también se registró con ambos métodos (6,6 puntos con la telefonía fija y 6,4 con la móvil).

\section{GRÁFICO 2 \\ ÍNDICE DE CONFIANZA DEL CONSUMIDOR POR TIPO DE TELEFONÍA SEGÚN MES DE LA ENCUESTA}

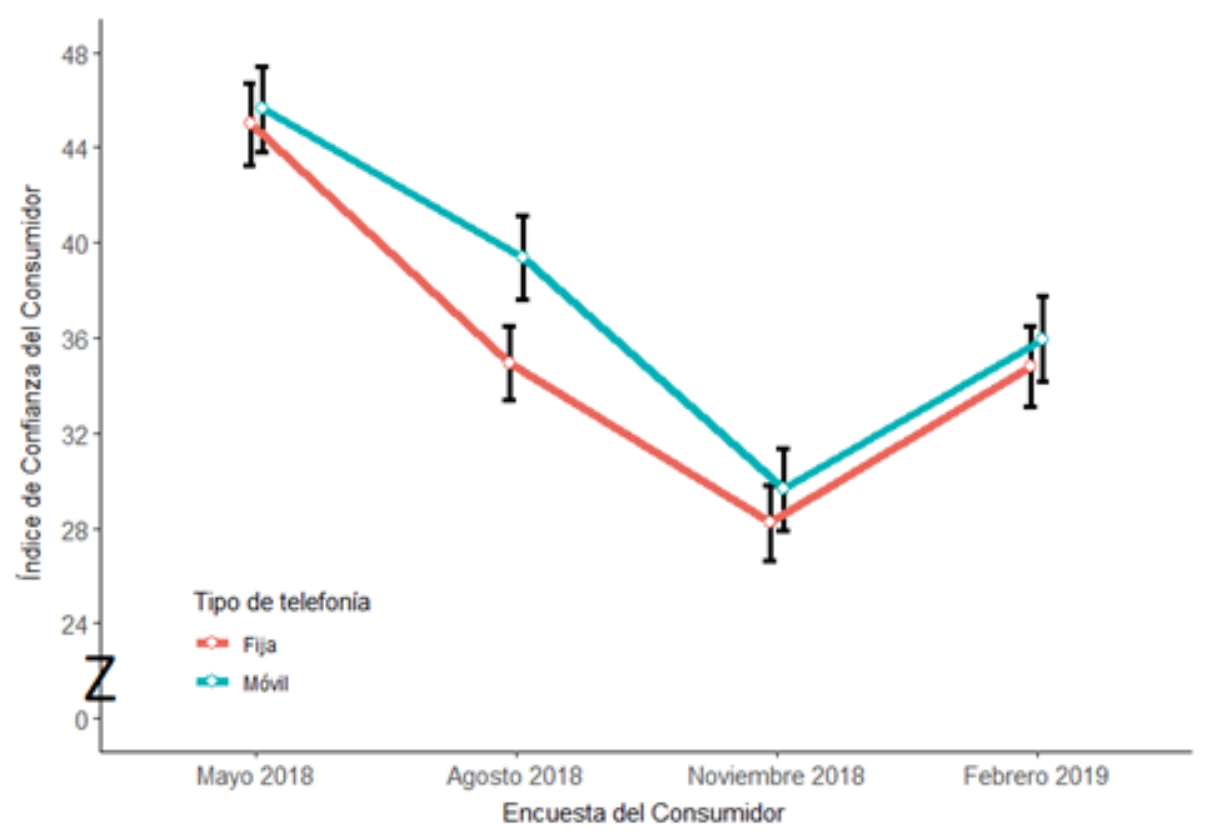

Fuente: : Elaboración propia a partir de los datos de la Escuela de Estadística, Universidad de Costa Rica (2019) 


\section{ÍNDICE DE CONFIANZA DEL CONSUMIDOR POR TIPO DE TELEFONÍA SEGÚN MES DE LA ENCUESTA}

\begin{tabular}{|c|c|c|c|c|c|}
\hline \multirow{2}{*}{ Mes } & \multicolumn{2}{|c|}{ Tipo de telefonía } & \multirow{2}{*}{$\begin{array}{l}\text { Diferencia } \\
\text { Fija-Móvil }\end{array}$} & \multirow{2}{*}{$\begin{array}{c}\text { Cambio } \\
\text { fija } \\
(\mathrm{t}+1-\mathrm{t})\end{array}$} & \multirow{2}{*}{$\begin{array}{c}\text { Cambio } \\
\text { Móvil } \\
(\mathrm{t}+1-\mathrm{t})\end{array}$} \\
\hline & Fija & Móvil & & & \\
\hline Mayo 2018 & 45,0 & 45,6 & $-0,6$ & & \\
\hline Agosto 2018 & 34,9 & 39,4 & $-4,4$ & $-10,1$ & $-6,2$ \\
\hline Noviembre 2018 & 28,2 & 29,6 & $-1,4$ & $-6,7$ & $-9,8$ \\
\hline Febrero 2018 & 34,8 & 36,0 & $-1,2$ & 6,6 & 6,4 \\
\hline Mayo 2019 & -- & 32,9 & 3,1 & -- & $-3,1$ \\
\hline
\end{tabular}

Fuente: Elaboración propia a partir de los datos de la Escuela de Estadística, Universidad de Costa Rica (2019)

La interrogante que origina el resultado anterior es si este se mantiene cuando el ICC se calcula para diferentes estratos poblacionales, establecidos por las características sociodemográficas del informante y las socioeconómicas de los hogares. En el cuadro 4, se optó mostrar los grupos extremos de las características sociodemográficas y, en el cuadro anexo 2, está disponible toda la información con los intervalos de confianza. Se observa que las discrepancias en las estimaciones del ICC entre ambos tipos de encuestas son menores a 5 puntos y se corrobora, calculando los intervalos de confianza, que no existen diferencias estadísticamente significativas (cuadro anexo 2). La excepción se encontró en agosto del 2018, para el caso de las mujeres (diferencia de -5,1 puntos entre la telefonía fija y móvil) y en febrero del 2019 para las personas de 50 años y más (diferencia de 5,4 puntos entre la telefonía fija y móvil). Esto revela que las estimaciones del ICC, según las variables sociodemográficas del informante, son similares entre ambos tipos de encuesta.

En el caso de las características socioeconómicas de los hogares, en el cuadro 5 se muestran algunas categorías relevantes y en el cuadro Anexo 3 está el detalle, incluyendo los intervalos de confianza. Como sucede con las características socioeconómicas, las estimaciones del ICC son bastante parecidas entre los diferentes subgrupos, pues los intervalos de confianza así lo corroboran. Las excepciones encontradas se presentan en agosto, para la capacidad de gasto discrecional en el hogar, tanto para quienes dicen tener la capacidad (diferencia de $-5,2$ puntos entre la telefonía fija y la móvil) como para los que no (diferencia de $-4,9$ puntos), cuando el ingreso mensual del hogar es menor a 500 mil colones mensuales (diferencia de $-7,2$ puntos) y entre quienes afirman no tener grandes dificultades con el ingreso mensual que reciben en el hogar (diferencia de -6,8 puntos). En todos los casos con diferencias significativas, la metodología con la telefonía móvil produce ICC más altos que con la fija. Sin embargo, más importante es que solo en 4 de las 32 comparaciones realizadas se encontraron diferencias, lo que sugiere bastante similitud de resultados entre ambas metodologías cuadro Anexo 3). 


\section{CUADRO 4 \\ ÍNDICE DE CONFIANZA DEL CONSUMIDOR PARA LAS CARACTERÍSTICAS SOCIODEMOGRÁFICAS DEL INFORMANTE POR TIPO DE TELEFONÍA SEGÚN MES DE LA ENCUESTA}

\begin{tabular}{|c|c|c|c|}
\hline \multirow[b]{2}{*}{ Características sociodemográficas } & \multicolumn{2}{|c|}{ Tipo de telefonía } & \multirow[b]{2}{*}{$\begin{array}{c}\text { Diferencia } \\
\text { (\%fija- \% móvil) }\end{array}$} \\
\hline & Fija & Móvil & \\
\hline \multicolumn{4}{|l|}{ SEXO } \\
\hline \multicolumn{4}{|l|}{ Hombre } \\
\hline Mayo 2018 & 47,2 & 45,9 & 1,3 \\
\hline Agosto 2018 & 35,0 & 38,8 & $-3,8$ \\
\hline Noviembre 2018 & 29,7 & 29,5 & 0,2 \\
\hline Febrero 2019 & 36,1 & 37,4 & $-1,3$ \\
\hline \multicolumn{4}{|l|}{ Mujer } \\
\hline Mayo 2018 & 42,8 & 45,4 & $-2,6$ \\
\hline Agosto 2018 & 34,8 & 39,9 & $-5,1$ \\
\hline Noviembre 2018 & 26,8 & 29,8 & $-3,0$ \\
\hline Febrero 2019 & 33,8 & 34,7 & $-0,9$ \\
\hline \multicolumn{4}{|l|}{ EDAD } \\
\hline \multicolumn{4}{|l|}{18 a 34 años } \\
\hline Mayo 2018 & 55,5 & 50,6 & 4,8 \\
\hline Agosto 2018 & 42,2 & 44,6 & $-2,4$ \\
\hline Noviembre 2018 & 30,5 & 34,8 & $-4,3$ \\
\hline Febrero 2019 & 43,5 & 42,0 & 1,4 \\
\hline \multicolumn{4}{|l|}{50 y más años } \\
\hline Mayo 2018 & 44,3 & 36,9 & 7,4 \\
\hline Agosto 2018 & 34,0 & 31,7 & 2,3 \\
\hline Noviembre 2018 & 28,0 & 21,5 & 6,4 \\
\hline Febrero 2019 & 33,3 & 28,0 & 5,4 \\
\hline \multicolumn{4}{|l|}{ EDUCACION } \\
\hline \multicolumn{4}{|l|}{ Primaria } \\
\hline Mayo 2018 & 45,0 & 45,6 & $-0,6$ \\
\hline Agosto 2018 & 34,9 & 39,4 & $-4,5$ \\
\hline Noviembre 2018 & 28,2 & 29,6 & $-1,4$ \\
\hline Febrero 2019 & 30,4 & 29,9 & 0,5 \\
\hline \multicolumn{4}{|l|}{ Universitaria } \\
\hline Mayo 2018 & 47,8 & 48,8 & $-1,0$ \\
\hline Agosto 2018 & 34,5 & 41,1 & $-6,6$ \\
\hline Noviembre 2018 & 28,6 & 31,9 & $-3,3$ \\
\hline Febrero 2019 & 38,2 & 41,5 & $-3,3$ \\
\hline
\end{tabular}

Fuente: Elaboración propia a partir de los datos de la Escuela de Estadística, Universidad de Costa Rica (2019) 


\section{CUADRO 5 \\ ÍNDICE DE CONFIANZA DEL CONSUMIDOR PARA LAS CARACTERÍSTICAS SOCIOECONÓMICAS DEL HOGAR POR TIPO DE TELEFONÍA SEGÚN MES DE LA ENCUESTA}

\begin{tabular}{|c|c|c|c|}
\hline \multirow{2}{*}{ Características sociodemográficas } & \multicolumn{2}{|c|}{ Tipo de telefonía } & \multirow[b]{2}{*}{ Diferencia } \\
\hline & Fija & Móvil & \\
\hline \multicolumn{4}{|l|}{ Con gasto discrecional } \\
\hline Mayo 2018 & 52,3 & 52,7 & $-0,4$ \\
\hline Agosto 2018 & 42,3 & 47,5 & $-5,2$ \\
\hline Noviembre 2018 & 34,4 & 39,0 & $-4,6$ \\
\hline Febrero 2019 & 41,6 & 45,6 & $-4,0$ \\
\hline \multicolumn{4}{|l|}{ Ingreso mensual de 500.000 o más } \\
\hline Mayo 2018 & 49,0 & 52,7 & $-3,7$ \\
\hline Agosto 2018 & 38,7 & 42,6 & $-3,9$ \\
\hline Noviembre 2018 & 30,8 & 35,0 & $-4,2$ \\
\hline Febrero 2019 & 39,6 & 43,5 & $-3,9$ \\
\hline \multicolumn{4}{|l|}{ INGRESO SUBJETIVO } \\
\hline \multicolumn{4}{|l|}{ Sin grandes dificultades } \\
\hline Mayo 2018 & 45,9 & 48,7 & $-2,7$ \\
\hline Agosto 2018 & 34,1 & 40,9 & $-6,8$ \\
\hline Noviembre 2018 & 28,3 & 30,7 & $-2,4$ \\
\hline Febrero 2019 & 34,7 & 36,5 & $-1,8$ \\
\hline \multicolumn{4}{|l|}{ Pueden ahorrar } \\
\hline Mayo 2018 & 55,5 & 57,6 & $-2,1$ \\
\hline Agosto 2018 & 44,7 & 51,5 & $-6,8$ \\
\hline Noviembre 2018 & 39,1 & 44,2 & $-5,1$ \\
\hline Febrero 2019 & 48,3 & 52,5 & $-4,2$ \\
\hline
\end{tabular}

Fuente: Fuente: Elaboración propia a partir de los datos de la Escuela de Estadística, Universidad de Costa Rica (2019) 


\section{Indicadores adicionales de interés}

Con base en el ICC, se clasifica por los consumidores en tres grupos: pesimistas, ambivalentes y optimistas. Esta clasificación forma parte de los informes de resultados que se publican trimestralmente sobre la encuesta y fue establecida usando análisis de conglomerados, procedimiento k-medias (Dillon \& Goldstein, 1984, p. 157- 205). Específicamente, para cada tipo de consumidor, se establece un ICC fijo: en los consumidores pesimistas es 13,6, en los ambivalentes es 43,1 y en los optimistas 77,9. Las distribuciones porcentuales de la clasificación muestran bastante similitud entre ambas metodologías, para las diferentes encuestas (gráfico 3). La excepción se presenta en agosto, pues los optimistas constituyen 7,6\% con la telefonía fija y 14,3\% con la móvil, diferencia que resultó estadísticamente significativa (cuadro Anexo 4).

\section{GRÁFICO 3 \\ DISTRIBUCIÓN PORCENTUAL DE LA CLASIFICACIÓN DE LOS CONSUMIDORES POR TIPO DE TELEFONÍA SEGÚN MES DE LA ENCUESTA}

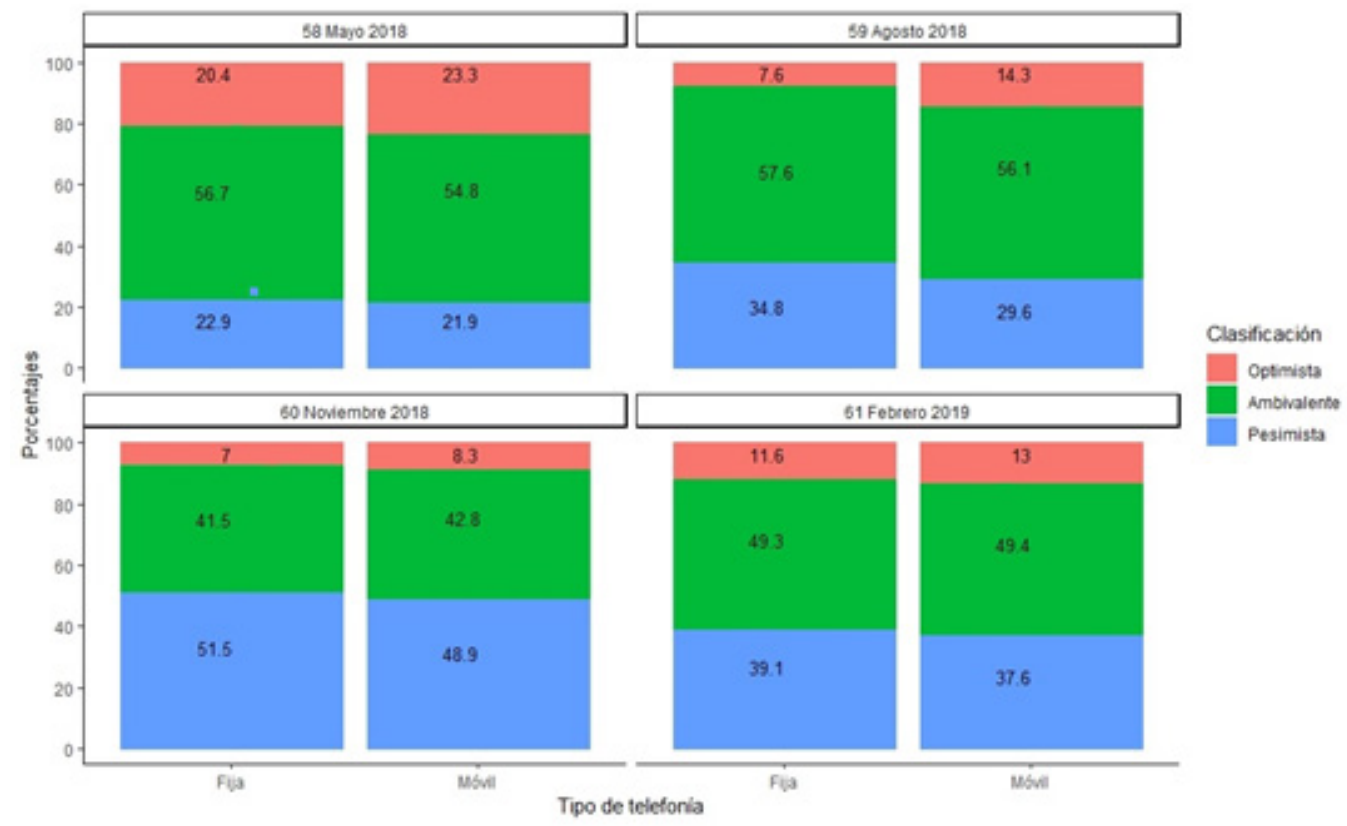

Nota: Para clasificar a los consumidores se utiliza la técnica de conglomerados (procedimiento de K-Medias). El objetivo es clasificarlos con base en la magnitud del ICC, agrupando consumidores que se asemejen en su confianza para lograr que entre los grupos se diferencien. La magnitud del ICC para cada grupo es fija y en el de consumidores pesimistas es 13,6, en los ambivalentes es 43,1 y en los optimistas de 77,9.

Fuente: Elaboración propia a partir de los datos de la Escuela de Estadística, Universidad de Costa Rica (2019)

Otros indicadores de interés que podrían presentar algún impacto con el cambio en la metodología son los que se derivan del ICC: el Índice de Condiciones Económicas Actuales (ICEA) y el Índice de Expectativas Económicas (IEE). La magnitud y tendencia de ambos es de interés, pues se trata de indicadores que contribuyen a conocer qué valoración están haciendo los consumidores del momento actual en el campo económico (ICEA) y también cuáles son sus expectativas sobre el futuro económico del país (IEE).

En el caso del ICEA, tanto la telefonía fija como la móvil revelan resultados similares y no se encontraron diferencias significativas entre ambos tipos de encuesta, para las diferentes mediciones 
(gráfico 4). En el caso del IEE, las estimaciones no revelan evidencia de ser diferentes, excepto en agosto del 2018, pues la diferencia entre la telefonía fija y la móvil resultó significativa (-6,6 puntos) (gráfico 4 y cuadro 6). Como sucede con el ICC, ambos índices, con ambas metodologías, presentan la misma tendencia en las cuatro encuestas. En el caso del ICEA, la disminución de mayo a noviembre de 2018, usando la telefonía fija, fue de -13,2 puntos y con la telefonía móvil fue de -14,6 puntos. El aumento que registró este mismo índice de noviembre de 2018 a febrero de 2019 fue de 5,7 puntos con la telefonía fija y en el mismo período fue de 7,6 puntos con la móvil.

\section{GRÁFICO 4 \\ COMPARACIÓN DE LA VALORACIÓN DE LAS CONDICIONES ECONÓMICAS ACTUALES (ICEA) Y DE LAS EXPECTATIVAS ECONÓMICAS (IEE) POR TIPO DE TELEFONÍA SEGÚN MES DE LA ENCUESTA}

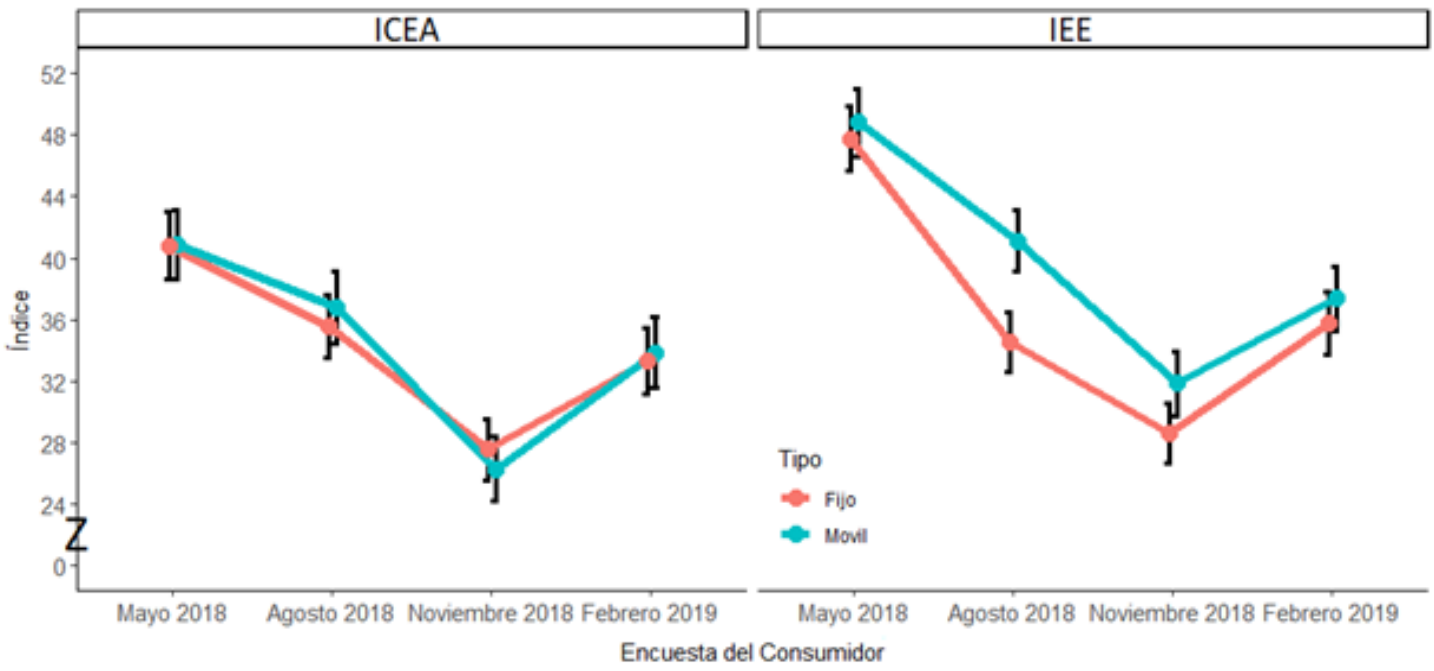

Fuente: Elaboración propia a partir de los datos de la Escuela de Estadística, Universidad de Costa Rica (2019)

\section{CUADRO 6}

ÍNDICE DE CONDICIONES ECONÓMICAS ACTUALES (ICEA) E ÍNDICE DE EXPECTATIVAS ECONÓMICAS (IEE) POR TIPO DE TELEFONÍA SEGÚN MES DE LA ENCUESTA

\begin{tabular}{|c|c|c|c|}
\hline \multirow{2}{*}{ Mes } & \multicolumn{2}{|c|}{ Tipo de telefonía } & \multirow[b]{2}{*}{ Diferencia } \\
\hline & Fija & Móvil & \\
\hline \multicolumn{4}{|l|}{ ICEA } \\
\hline Mayo & 40,8 & 40,9 & $-0,1$ \\
\hline Agosto & 35,6 & 36,8 & $-1,2$ \\
\hline Noviembre & 27,6 & 26,3 & 1,3 \\
\hline Febrero & 33,3 & 33,9 & $-0,5$ \\
\hline \multicolumn{4}{|l|}{ IEE } \\
\hline Mayo & 47,8 & 48,8 & $-1,0$ \\
\hline Agosto & 34,5 & 41,1 & $-6,6$ \\
\hline Noviembre & 28,6 & 31,9 & $-3,2$ \\
\hline Febrero & 35,8 & 37,4 & $-1,6$ \\
\hline
\end{tabular}

Fuente: Elaboración propia a partir de los datos de la Escuela de Estadística, Universidad de Costa Rica (2019) 
El análisis del IEE revela un comportamiento similar, pues la disminución observada de mayo a noviembre de 2018 fue de -19,2 puntos con la telefonía fija y de -16,9 puntos con la móvil (gráfico 4 y cuadro 6). Además, el aumento observado de noviembre del 2018 a febrero del 2019 fue de 7,2 puntos con la telefonía fija y de 5,5 puntos con la móvil.

\section{Cobertura de la muestra de telefonía móvil}

Un último aspecto indispensable por revisar es que, al cambiar el tipo de telefonía, de la fija a la móvil, se pierde la capacidad de estratificar geográficamente, lo que invita a investigar la dispersión que puede tener una muestra de 700 usuarios de telefonía celular en todo el país. Esto puede estudiarse posestratificando geográficamente la muestra en cada encuesta, lo que es posible porque en el cuestionario con telefonía celular se solicitó a cada persona entrevistada su provincia, cantón y distrito de residencia.

Para realizar esta prueba se optó, como primera aproximación, comparar la distribución porcentual de las personas de 18 años y más por provincias con la que proviene del censo 2011. Es claro que esta comparación está afectada por el tiempo transcurrido y porque del censo se están seleccionando las personas de 18 años y más, usen o no celular. Esta decisión se tomó porque en ese año censal la tenencia de celular en los hogares era menor que la actual, pero tomando en cuenta que la distribución por provincia de las personas de 18 años y más cambia poco en el tiempo y que actualmente casi el 100\% de ese grupo de edad usa celular, se decidió hacerla.

Los resultados muestran que las distribuciones porcentuales del censo 2011 y la ENCON son bastante parecidas para las provincias, pues la mayor diferencia absoluta registrada es de 2,3 puntos porcentuales en Limón. Aunque la figura 1 solamente presenta la encuesta de noviembre de 2018, en el cuadro Anexo 6, se muestran las distribuciones por provincias de las cuatro encuestas. En general, se observa que la muestra de 700 usuarios de telefonía celular en Costa Rica presenta una distribución por provincia acorde con la derivada del censo 2011 y la diferencia porcentual más alta encontrada, con respecto al censo, en todas las encuestas fue de 3,8 puntos en febrero de 2019, en la provincia de Limón.

Una aproximación más acertada de esta situación se obtiene usando las regiones de planificación de la ENAHO 2018. De esta encuesta, se obtiene la distribución de las personas de 18 años o más en cuyo hogar poseen al menos un celular para compararla con la ENCON y las similitudes entre ambas distribuciones son claras. La mayor diferencia absoluta encontrada es de 5,7 puntos porcentuales en la Región Central. Si bien la figura 2 muestra únicamente el resultado para el mes de noviembre de 2018, en la tabla Anexo 7 están las distribuciones porcentuales de las cuatro encuestas. Con base en esa información, se observa cómo la Región Central generalmente presenta más diferencias en todas las encuestas con respecto a la Encuesta Nacional de Hogares 2018 y llega hasta 8,2 puntos en febrero de 2019. 


\section{FIGURA 1}

COMPARACIÓN POR PROVINCIAS DE LAS DISTRIBUCIONES PORCENTUALES DE LAS DE PERSONAS DE 18 AÑOS Y MÁS DEL CENSO NACIONAL DE POBLACIÓN Y VIVIENDA 2011 Y LA ENCUESTA DE CONFIANZA DEL CONSUMIDOR DE NOVIEMBRE DEL 2018

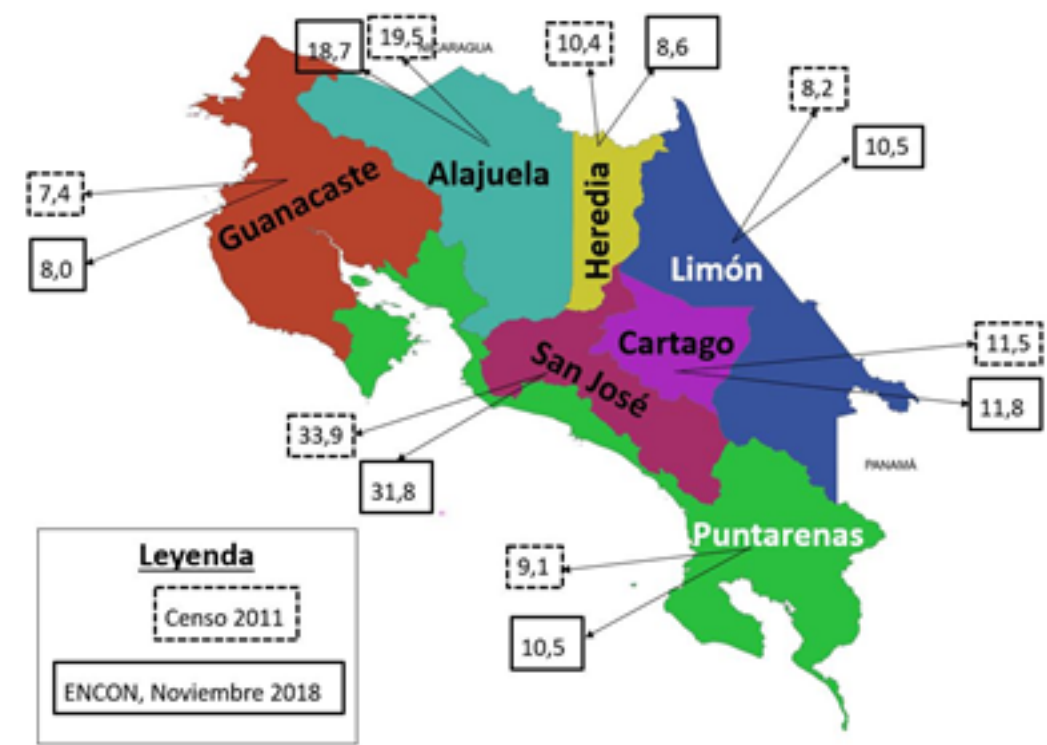

Fuente: Elaboración propia a partir de los datos de la Escuela de Estadística, Universidad de Costa Rica (2019) y a partir de los datos de Instituto Nacional de Estadística y Censos (2012).

FIGURA 2

COMPARACIÓN DE LAS DISTRIBUCIONES PORCENTUALES DE LAS PERSONAS DE 18 AÑOS Y MÁS DE LA ENCUESTA NACIONAL DE HOGARES 2018 Y LA ENCUESTA DE CONFIANZA DE LOS CONSUMIDORES DE NOVIEMBRE DEL 2018

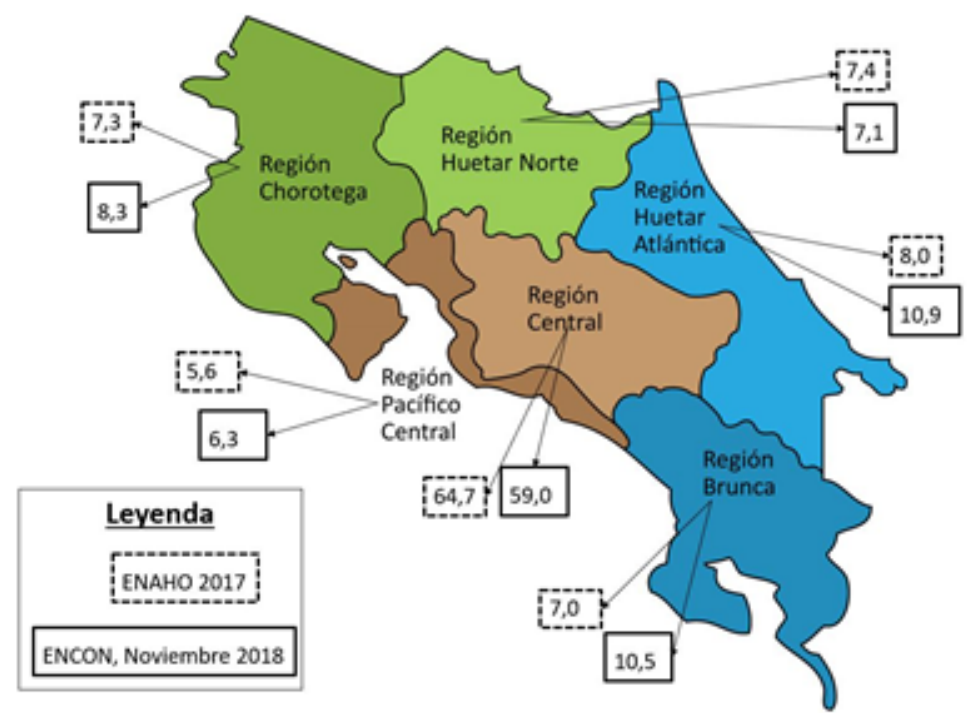

Fuente: Elaboración propia a partir de los datos de la Escuela de Estadística, Universidad de Costa Rica (2019) y a partir de los datos de Instituto Nacional de Estadística y Censos (2012). 


\section{CONCLUSIONES}

El modificar la metodología de la Encuesta de Confianza del Consumidor, la que tradicionalmente se hace usando telefonía fija (residencial) y entrevistando al principal sostén económico del hogar, por otra en la que se usa telefonía móvil (celular) y entrevistando a una persona de 18 años y más que usa celular, no altera, estadísticamente hablando, la magnitud ni la tendencia del ICC, ni la de otros indicadores de interés, como el ICEA y el IEE. En otras palabras, la telefonía móvil produce estimaciones casi idénticas (excepto en agosto de 2018) a las obtenidas con la telefonía fija $y$, además, la tendencia de los indicadores más importantes es prácticamente la misma. Esto significa que las valoraciones y expectativas de los consumidores en el campo económico, con ambos tipos de encuestas, son similares.

El resultado de esta investigación es favorecido por la ejecución de cuatro encuestas y no solamente una, lo que genera más confianza para afirmar que puede continuarse utilizando la serie de datos de las 61 mediciones disponibles. A esto, debe sumársele el que, con el cambio metodológico, la cobertura geográfica perdida por utilizar la telefonía fija ahora se fortalece, abarcando el ámbito nacional.

La similitud observada entre los resultados obtenidos con ambas metodologías se produce, pese a que los informantes revelaron características sociodemográficas y socioeconómicas diferentes. Específicamente, la encuesta con telefonía fija, cuyo informante es el principal sostén económico del hogar, representa, predominantemente, personas de 50 años o más (promedio de $65 \%$ en las cuatro encuestas), con educación universitaria (promedio de 44\%); mientras que la realizada con telefonía móvil, cuyo informante es una persona de 18 años o más que usa celular, representa, principalmente, personas menores a 50 años (promedio de 64\%) y niveles educativos de primaria y secundaria (promedio de 78\%).

En este tema del informante $-y$ las diferencias que presenta-, debe tomarse en cuenta que también entre los usuarios de la telefonía móvil existen quienes son principal sostén económico del hogar. Una tabulación adicional de las cuatro encuestas con telefonía móvil muestra que el porcentaje de personas de 18 años y más, usuarias de celular y que desarrollan el rol de principal sostén económico del hogar, está entre 55\% y 61\%. En otras palabras, aproximadamente 58\% de los informantes de la telefonía móvil (en promedio) también constituye el principal sostén económico del hogar, lo que contribuye a explicar por qué las mediciones obtenidas a partir de ambas metodologías son tan parecidas.

Finalmente, un resultado favorable es corroborar que una muestra de 700 entrevistas, con la nueva metodología, produce una dispersión geográfica que garantiza la inclusión de consumidores de todo el país, en términos casi proporcionales a grandes áreas geográficas, como las provincias y las regiones de planificación. Con la metodología anterior, era posible estratificar geográficamente, porque la telefonía fija lo permitía, dado que las centrales telefónicas activas proveen una ubicación geográfica, aproximada, de los hogares en los cantones de residencia. La ventaja de aplicar este procedimiento es que existía garantía de asignar proporcionalmente la muestra telefónica en al menos tres zonas del país, lo que mejoraba el procedimiento de selección de teléfonos.

Al cambiar la metodología, usando ahora telefonía móvil, se perdió la posibilidad de estratificar geográficamente, pues ni la asignación ni el uso del dispositivo están sujetos a una ubicación geográfica determinada de residencia. No obstante, es claro que una posestratificación corrobora que el tamaño de muestra y el procedimiento para seleccionarla garantiza una dispersión apropiada en todo el país. Esto no significa que la encuesta tiene la capacidad para realizar estimaciones en esos ámbitos geográficos, sino que la dispersión de la muestra de telefonía móvil en Costa Rica es adecuada para los fines propuestos, lo que puede continuar evaluándose en las encuestas futuras, pues se continuará recolectando la información geográfica correspondiente. 
Con el cambio de metodología realizado, la ENCON acoge las recomendaciones emitidas en el Código de Buenas Prácticas Estadísticas en Costa Rica promovido por el Sistema de Estadística Nacional (SEN) y el Instituto Nacional de Estadística y Censos (INEC) (Instituto Nacional de Estadística y Censos, 2014). En este sentido, la investigación realizada responde al principio de poseer una metodología sólida, que se evalúa periódicamente y se ajusta para garantizar la calidad de los productos estadísticos (p. 10). También se relaciona con el principio de poseer procesos estadísticos adecuados, desarrollando revisiones de la encuesta y dando a conocer los resultados públicamente (p.10). El principio de precisión y fiabilidad que recomienda el SEN también es abordado, pues la revisión de la metodología de la ENCON tuvo como meta mejorar la calidad de las estadísticas, lo que se logró comparando las telefonías disponibles y ampliando la cobertura de la muestra en el país (p. 14). La revisión de la metodología y los cambios ejecutados se fortalecen al saber que, según un grupo de expertos liderados por Paul J. Lavrakas, la telefonía móvil seguirá siendo en el futuro un medio importante para realizar encuestas por muestreo, pues proporciona una amplia cobertura (disminuye el error de cobertura) y pueden aplicarse métodos para ajustar los sesgos que aparezcan (Lavrakas et al., 2017). Es decir, con el cambio metodológico realizado, la ENCON continuará proporcionando información oportuna y de calidad en los próximos años.

\section{AGRADECIMIENTOS}

Se agradece al Director de la Escuela de Estadística, M.Sc. Fernando Ramírez Hernández, por apoyar la investigación realizada. También se agradece a la Unidad de Servicios Estadísticos (USES), de la Escuela de Estadística, particularmente a la Bach. Yorleny Quirós y al Bach. Moisés Coto, por coordinar de la recolección de los datos con las dos metodologías usadas.

\section{REFERENCIAS}

Dillon, W. R., \& Goldstein, M. (1984). Multivariate Analysis: Methods and Applications (4a ed.). New York: Wiley.

Escuela de Estadística, Universidad de Costa Rica (2017, 13 de diciembre). Curso Diseño y Ejecución de Encuestas: Encuesta Actualidades 2017. https://web.archive.org/web/20200421194032/ http://www.estadistica.ucr.ac.cr/index.php/es/encuestas/actualidades/informes/73-2017estadistica-ucr-actualidades/file

Escuela de Estadística, Universidad de Costa Rica. (2019). Rediseño de la Encuesta del Consumidor Mayo 2018 - Febrero 2019. [Fichero Digital]. San José, C.R

Foody, G. M. (2009). Classification accuracy comparison: hypothesis tests and the use of confidence intervals in evaluations of difference, equivalence and non-inferiority. Remote Sensing of Environment, 113(8), 1658-1663. https://doi.org/10.1016/j.rse.2009.03.014

Gardner, M. J., \& Altman, D. G. (1986). Confidence intervals rather than P values: estimation rather than hypothesis testing. Br Med J (Clin Res Ed), 292(6522), 746-750. https://doi.org/10.1136/ bmj.292.6522.746

Instituto Nacional de Estadística y Censos. (2014). Código de buenas prácticas estadístcas para Costa Rica. https://web.archive.org/web/20200421194538/http://sen.inec.cr/sites/default/files/ Documentos_NT/codigo_de_buenas_practicas.pdf

Instituto Nacional de Estadística y Censos. (2003). Encuesta de Propósitos Múltiples 2002. [Fichero Digital]. San José, C.R.: INEC. 2003.

Instituto Nacional de Estadística y Censos. (2004). Encuesta de Propósitos Múltiples 2003. [Fichero Digital]. San José, C.R.: INEC. 2004. 
Instituto Nacional de Estadística y Censos. (2005). Encuesta de Propósitos Múltiples 2004. [Fichero Digital]. San José, C.R.: INEC. 2005.

Instituto Nacional de Estadística y Censos. (2006). Encuesta de Propósitos Múltiples 2005. [Fichero Digital]. San José, C.R.: INEC. 2006.

Instituto Nacional de Estadística y Censos. (2007). Encuesta de Propósitos Múltiples 2006. [Fichero Digital]. San José, C.R.: INEC. 2007.

Instituto Nacional de Estadística y Censos. (2008). Encuesta de Propósitos Múltiples 2007. [Fichero Digital]. San José, C.R.: INEC. 2008.

Instituto Nacional de Estadística y Censos. (2009). Encuesta de Propósitos Múltiples 2008. [Fichero Digital]. San José, C.R.: INEC. 2009.

Instituto Nacional de Estadística y Censos. (2010). Encuesta de Propósitos Múltiples 2009. [Fichero Digital]. San José, C.R.: INEC. 2010.

Instituto Nacional de Estadística y Censos. (2011). Encuesta Nacional de Hogares 2010. [Fichero Digital]. San José, C.R.: INEC. 2011.

Instituto Nacional de Estadística y Censos. (2012). Encuesta Nacional de Hogares 2011. [Fichero Digital]. San José, C.R.: INEC. 2012.

Instituto Nacional de Estadística y Censos. (2013). Encuesta Nacional de Hogares 2012. [Fichero Digital]. San José, C.R.: INEC. 2013.

Instituto Nacional de Estadística y Censos. (2014). Encuesta Nacional de Hogares 2013. [Fichero Digital]. San José, C.R.: INEC. 2014.

Instituto Nacional de Estadística y Censos. (2015). Encuesta Nacional de Hogares 2014. [Fichero Digital]. San José, C.R.: INEC. 2015.

Instituto Nacional de Estadística y Censos. (2016). Encuesta Nacional de Hogares 2015. [Fichero Digital]. San José, C.R.: INEC. 2016.

Instituto Nacional de Estadística y Censos. (2017). Encuesta Nacional de Hogares 2016. [Fichero Digital]. San José, C.R.: INEC. 2017.

Instituto Nacional de Estadística y Censos. (2018). Encuesta Nacional de Hogares 2017. [Fichero Digital]. San José, C.R.: INEC. 2018.

Instituto Nacional de Estadística y Censos. (2018). Plan Estadístico Nacional (PEN) 2018-2022. San José, C.R.: INEC. 2018.

Instituto Nacional de Estadística y Censos. (2019). Encuesta Nacional de Hogares 2018. [Fichero Digital]. San José, C.R.: INEC. 2019.

Instituto Nacional de Estadística y Censos. (2012). X Censo Nacional de Población y VI de Vivienda, 2011. [Fichero Digital]. San José, C.R.: INEC. 2018.

Instituto Nacional de Estadística y Censos, Ministerio de Cultura y Juventud. Encuesta Nacional de Cultura 2016. (2017). Principales Resultados. Ed 1. San José, Costa Rica.

Katona, G. (1964). The mass consumption society. McGraw-Hill.

Lavrakas, P., et al. (2017). The Future Of U.S. General Population Telephone Survey Research. https://web.archive.org/web/20200421212817/https://www.aapor.org/Education-Resources/ Reports/The-Future-Of-U-S-General-Population-Telephone-Sur.aspx

Madrigal, Johnny. (2012). Encuesta de Confianza de los Consumidores. Metodolología de la Encuesta. San José, Costa Rica: Escuela de Estadística. https:/web.archive.org/ web/20200421213429/http://estadistica.ucr.ac.cr/index.php/es/encuestas/confianza-de-losconsumidores/metodología-de-encuesta-icc-n57/121-metodologia-encuesta-consumidor/file 
Richard T. Curtin. (1982). Indicator of Consumer Behavior: The University of Michigan Suverys of Consumers. Public Opinion Quarterly. 46(3), 340-35. https://doi.org/10.1086/268731

Waksberg, J. (1978). Sampling Methods for Random Digit Dialing. Journal of the American Statistical Association, 73(361), 40-46. https://doi.org/10.1080/01621459.1978.10479995

VII. ANEXOS

\section{ANEXO 1 \\ DISTRIBUCIONES PORCENTUALES DE LAS VARIABLES USADAS PARA CONSTRUIR EL ÍNDICE DE CONFIANZA DEL CONSUMIDOR POR TIPO DE TELEFONÍA SEGÚN MES DE LA ENCUESTA}

\begin{tabular}{|c|c|c|c|c|c|c|c|c|}
\hline \multirow{2}{*}{ Variables } & \multicolumn{2}{|c|}{ Mayo 2018} & \multicolumn{2}{|c|}{ Agosto 2018} & \multicolumn{2}{|c|}{ Noviembre 2018} & \multicolumn{2}{|c|}{ Febrero 2019} \\
\hline & Fija & Móvil & Fija & Móvil & Fija & Móvil & Fija & Móvil \\
\hline \multicolumn{9}{|l|}{$\begin{array}{l}\text { Situación económica } \\
\text { actual familiar }\end{array}$} \\
\hline Mejor & 15,0 & 21,3 & 10,7 & 17,3 & 11,1 & 14,3 & 14,0 & 18,1 \\
\hline Igual & 51,6 & 46,1 & 53,2 & 43,8 & 44,8 & 39,9 & 47,1 & 41,5 \\
\hline Peor ahora & 33,1 & 32,0 & 35,4 & 38,7 & 44,1 & 45,6 & 38,3 & 39,9 \\
\hline No sabe & 0,3 & 0,6 & 0,7 & 0,1 & 0,0 & 0,1 & 0,6 & 0,6 \\
\hline \multicolumn{9}{|c|}{$\begin{array}{l}\text { Momento para adquirir } \\
\text { artículos grandes }\end{array}$} \\
\hline Buenos tiempos & 27,7 & 25,3 & 20,4 & 21,4 & 13,7 & 11,4 & 17,6 & 17,8 \\
\hline Ni uno ni otro & 23,6 & 20,9 & 23,0 & 24,5 & 15,4 & 13,1 & 18,1 & 19,3 \\
\hline Malos tiempos & 46,3 & 51,0 & 53,5 & 52,8 & 70,5 & 75,0 & 60,3 & 60,6 \\
\hline No sabe & 2,4 & 2,9 & 3,1 & 1,3 & 0,4 & 0,6 & 4,0 & 2,4 \\
\hline \multicolumn{9}{|l|}{$\begin{array}{l}\text { Situación económica } \\
\text { futura familiar }\end{array}$} \\
\hline Mejor & 30,3 & 36,7 & 16,8 & 28,7 & 18,3 & 24,9 & 23,9 & 31,0 \\
\hline Igual & 35,1 & 30,6 & 39,6 & 39,3 & 31,8 & 27,2 & 35,7 & 28,8 \\
\hline Peor & 25,6 & 24,1 & 34,9 & 26,0 & 49,5 & 43,0 & 37,6 & 35,0 \\
\hline No sabe & 9,0 & 8,6 & 8,7 & 6,1 & 0,4 & 4,9 & 2,9 & 5,2 \\
\hline \multicolumn{9}{|l|}{$\begin{array}{l}\text { Situación futura } \\
\text { de las empresas }\end{array}$} \\
\hline Condiciones favorables & 7,3 & 10,3 & 3,1 & 9,1 & 3,7 & 6,3 & 5,9 & 5,9 \\
\hline Buenas con reservas & 23,1 & 18,5 & 11,0 & 13,9 & 7,7 & 7,9 & 11,7 & 11,0 \\
\hline Ni uno ni otro & 32,0 & 35,8 & 28,5 & 29,9 & 24,8 & 25,8 & 26,1 & 28,4 \\
\hline Malas con reservas & 18,3 & 16,2 & 30,0 & 22,7 & 30,0 & 23,4 & 30,3 & 26,0 \\
\hline Condiciones desfavo. & 12,1 & 11,9 & 20,3 & 19,9 & 29,5 & 31,6 & 20,3 & 25,3 \\
\hline No sabe & 7,1 & 7,4 & 7,2 & 4,5 & 4,3 & 5,0 & 5,7 & 3,4 \\
\hline
\end{tabular}




\begin{tabular}{|c|c|c|c|c|c|c|c|c|}
\hline \multirow{2}{*}{ Variables } & \multicolumn{2}{|c|}{ Mayo 2018} & \multicolumn{2}{|c|}{ Agosto 2018} & \multicolumn{2}{|c|}{ Noviembre 2018} & \multicolumn{2}{|c|}{ Febrero 2019} \\
\hline & Fija & Móvil & Fija & Móvil & Fija & Móvil & Fija & Móvil \\
\hline \multicolumn{9}{|c|}{$\begin{array}{l}\text { Situación del país en } \\
5 \text { años }\end{array}$} \\
\hline Buenos tiempos & 29,0 & 27,3 & 18,2 & 16,0 & 15,5 & 13,6 & 20,9 & 18,3 \\
\hline Ni uno ni otro & 18,1 & 19,0 & 17,9 & 27,2 & 18,1 & 18,1 & 13,6 & 21,0 \\
\hline Tiempos de crisis & 47,1 & 47,8 & 56,6 & 52,3 & 64,3 & 63,3 & 59,1 & 55,7 \\
\hline No sabe & 5,7 & 5,9 & 7,3 & 4,4 & 2,0 & 4,9 & 6,4 & 5,0 \\
\hline
\end{tabular}

Fuente: Elaboración propia a partir de los datos de la Escuela de Estadística, Universidad de Costa Rica (2019)

\section{ANEXO 2 \\ INTERVALOS DE CONFIANZA DEL ÍNDICE DE CONFIANZA DEL CONSUMIDOR PARA LAS CARACTERÍSTICAS SOCIODEMOGRÁFICAS POR TIPO DE TELEFONÍA SEGÚN MES DE LA ENCUESTA}

\begin{tabular}{|c|c|c|c|c|c|c|}
\hline \multirow{3}{*}{$\begin{array}{l}\text { Características } \\
\text { sociodemográficas }\end{array}$} & \multicolumn{6}{|c|}{ Tipo de telefonía } \\
\hline & \multicolumn{3}{|c|}{ Fija } & \multicolumn{3}{|c|}{ Móvil } \\
\hline & Inferior & Promedio & Superior & Inferior & Promedio & Superior \\
\hline SEXO & 100,00 & 100,00 & 100,00 & 100,00 & 100,00 & 100,00 \\
\hline \multicolumn{7}{|l|}{ Hombre } \\
\hline Mayo 2018 & 44,7 & 47,2 & 49,6 & 43,2 & 45,9 & 48,6 \\
\hline Agosto 2018 & 32,7 & 35,0 & 37,3 & 36,2 & 38,8 & 41,4 \\
\hline Noviembre 2018 & 27,2 & 29,7 & 32,2 & 26,9 & 29,5 & 32,1 \\
\hline Febrero 2019 & 33,2 & 36,1 & 38,9 & 34,7 & 37,4 & 40,1 \\
\hline \multicolumn{7}{|l|}{ Mujer } \\
\hline Mayo 2018 & 40,3 & 42,8 & 45,2 & 42,9 & 45,4 & 47,8 \\
\hline Agosto 2018 & 32,6 & 34,8 & 37,0 & 37,6 & 39,9 & 42,2 \\
\hline Noviembre 2018 & 24,7 & 26,8 & 28,9 & 27,4 & 29,8 & 32,1 \\
\hline Febrero 2019 & 31,7 & 33,8 & 35,9 & 32,2 & 34,7 & 37,1 \\
\hline \multirow{2}{*}{\multicolumn{7}{|c|}{$\begin{array}{l}\text { EDAD } \\
18 \text { a } 34 \text { años }\end{array}$}} \\
\hline & & & & & & \\
\hline Mayo 2018 & 49,0 & 55,5 & 61,9 & 48,1 & 50,6 & 53,2 \\
\hline Agosto 2018 & 36,5 & 42,2 & 47,9 & 41,9 & 44,6 & 47,4 \\
\hline Noviembre 2018 & 25,5 & 30,5 & 35,5 & 32,1 & 34,8 & 37,6 \\
\hline Febrero 2019 & 38,2 & 43,5 & 48,8 & 39,3 & 42,0 & 44,8 \\
\hline \multicolumn{7}{|l|}{34 a 50 años } \\
\hline Mayo 2018 & 40,1 & 43,6 & 47,1 & 42,3 & 45,8 & 49,3 \\
\hline Agosto 2018 & 31,1 & 34,3 & 37,5 & 35,0 & 38,2 & 41,4 \\
\hline Noviembre 2018 & 24,9 & 28,4 & 31,8 & 25,9 & 28,9 & 31,9 \\
\hline Febrero 2019 & 31,4 & 35,3 & 39,2 & 35,2 & 38,4 & 41,1 \\
\hline
\end{tabular}




\begin{tabular}{|c|c|c|c|c|c|c|}
\hline \multirow{3}{*}{$\begin{array}{l}\text { Características } \\
\text { sociodemográficas }\end{array}$} & \multicolumn{6}{|c|}{ Tipo de telefonía } \\
\hline & \multicolumn{3}{|c|}{ Fija } & \multicolumn{3}{|c|}{ Móvil } \\
\hline & Inferior & Promedio & Superior & Inferior & Promedio & Superior \\
\hline \multicolumn{7}{|l|}{50 y más años } \\
\hline Mayo 2018 & 42,2 & 44,3 & 46,4 & 36,9 & 36,9 & 43,5 \\
\hline Agosto 2018 & 32,1 & 34,0 & 35,9 & 31,7 & 31,7 & 37,7 \\
\hline Noviembre 2018 & 26,0 & 28,0 & 29,9 & 21,5 & 21,5 & 27,7 \\
\hline Febrero 2019 & 31,3 & 33,3 & 35,3 & 24,9 & 28,0 & 31,1 \\
\hline \multicolumn{7}{|l|}{ EDUCACIÓN } \\
\hline \multicolumn{7}{|l|}{ Primaria } \\
\hline Mayo 2018 & 39,5 & 45,0 & 45,3 & 37,8 & 45,6 & 43,9 \\
\hline Agosto 2018 & 30,0 & 34,9 & 34,9 & 34,1 & 39,4 & 40,5 \\
\hline Noviembre 2018 & 23,8 & 28,2 & 29,6 & 22,1 & 29,6 & 28,2 \\
\hline Febrero 2019 & 27,4 & 30,4 & 33,4 & 26,8 & 29,9 & 33,1 \\
\hline \multicolumn{7}{|l|}{ Secundaria } \\
\hline Mayo 2018 & 40,6 & 40,8 & 47,3 & 45,0 & 40,9 & 50,8 \\
\hline Agosto 2018 & 34,0 & 35,6 & 41,3 & 38,3 & 36,8 & 43,5 \\
\hline Noviembre 2018 & 26,8 & 27,6 & 33,5 & 29,5 & 26,3 & 35,1 \\
\hline Febrero 2019 & 31,4 & 34,4 & 37,4 & 36,2 & 38,9 & 41,6 \\
\hline \multicolumn{7}{|l|}{ Universitaria } \\
\hline Mayo 2018 & 44,6 & 47,8 & 50,2 & 47,1 & 48,8 & 53,4 \\
\hline Agosto 2018 & 33,1 & 34,5 & 37,9 & 37,7 & 41,1 & 43,6 \\
\hline Noviembre 2018 & 25,9 & 28,6 & 30,7 & 30,4 & 31,9 & 35,9 \\
\hline Febrero 2019 & 35,5 & 38,2 & 41,0 & 38,2 & 41,5 & 44,8 \\
\hline
\end{tabular}

Fuente: Elaboración propia a partir de los datos de la Escuela de Estadística, Universidad de Costa Rica (2019)

$$
\begin{gathered}
\text { ANEXO } 3 \\
\text { INTERVALOS DE CONFIANZA DEL ÍNDICE DE CONFIANZA DEL CONSUMIDOR PARA } \\
\text { LAS CARACTERÍSTICAS SOCIOECONÓMICAS DEL HOGAR } \\
\text { POR TIPO DE TELEFONÍA SEGÚN MES DE LA ENCUESTA }
\end{gathered}
$$

\begin{tabular}{|c|c|c|c|c|c|c|}
\hline \multirow{3}{*}{$\begin{array}{l}\text { Características } \\
\text { sociodemográficas }\end{array}$} & \multicolumn{6}{|c|}{ Tipo de telefonía } \\
\hline & \multicolumn{3}{|c|}{ Fija } & \multicolumn{3}{|c|}{ Móvil } \\
\hline & Inferior & Promedio & Superior & Inferior & Promedio & Superior \\
\hline \multicolumn{7}{|c|}{$\begin{array}{l}\text { GASTO DISCRECIONAL } \\
\text { No }\end{array}$} \\
\hline Mayo 2018 & 35,4 & 37,6 & 39,8 & 39,0 & 41,2 & 43,5 \\
\hline Agosto 2018 & 27,8 & 29,7 & 31,5 & 32,6 & 34,6 & 36,7 \\
\hline Noviembre 2018 & 21,7 & 23,4 & 25,4 & 23,5 & 25,4 & 27,4 \\
\hline Febrero 2019 & 27,0 & 29,1 & 31,2 & 28,9 & 31,0 & 33,2 \\
\hline
\end{tabular}




\begin{tabular}{|c|c|c|c|c|c|c|}
\hline \multirow{3}{*}{$\begin{array}{l}\text { Características } \\
\text { sociodemográficas }\end{array}$} & \multicolumn{6}{|c|}{ Tipo de telefonía } \\
\hline & \multicolumn{3}{|c|}{ Fija } & \multicolumn{3}{|c|}{ Móvil } \\
\hline & Inferior & Promedio & Superior & Inferior & Promedio & Superior \\
\hline \multicolumn{7}{|l|}{ Si } \\
\hline Mayo 2018 & 49,8 & 52,3 & 54,8 & 49,9 & 52,7 & 55,6 \\
\hline Agosto 2018 & 39,8 & 42,3 & 44,8 & 44,7 & 47,5 & 50,4 \\
\hline Noviembre 2018 & 31,8 & 34,4 & 37,2 & 35,9 & 39,0 & 42,1 \\
\hline Febrero 2019 & 38,9 & 41,6 & 44,2 & 42,5 & 45,6 & 48,8 \\
\hline \multicolumn{7}{|l|}{ INGRESO MENSUAL } \\
\hline \multicolumn{7}{|l|}{ Menos de 500.000} \\
\hline Mayo 2018 & 38,0 & 40,4 & 42,8 & 39,3 & 41,6 & 43,9 \\
\hline Agosto 2018 & 28,6 & 30,7 & 32,9 & 35,7 & 37,9 & 40,2 \\
\hline Noviembre 2018 & 23,0 & 25,1 & 27,1 & 24,4 & 26,7 & 28,9 \\
\hline Febrero 2019 & 27,0 & 29,1 & 31,3 & 30,2 & 32,3 & 34,5 \\
\hline \multicolumn{7}{|l|}{500.000 o más } \\
\hline Mayo 2018 & 46,3 & 49,0 & 51,6 & 49,9 & 52,7 & 55,5 \\
\hline Agosto 2018 & 36,3 & 38,7 & 41,1 & 39,5 & 42,6 & 45,7 \\
\hline Noviembre 2018 & 28,3 & 30,8 & 33,3 & 32,3 & 35,0 & 37,7 \\
\hline Febrero 2019 & 36,9 & 39,6 & 42,2 & 40,4 & 43,5 & 46,6 \\
\hline \multirow{2}{*}{\multicolumn{7}{|c|}{$\begin{array}{l}\text { INGRESO SUBJETIVO } \\
\text { Tienen grandes dificultades }\end{array}$}} \\
\hline & & & & & & \\
\hline Mayo 2018 & 25,0 & 30,0 & 35,0 & 25,6 & 30,8 & 35,9 \\
\hline Agosto 2018 & 17,6 & 23,6 & 29,5 & 25,6 & 30,9 & 36,2 \\
\hline Noviembre 2018 & 13,2 & 17,1 & 21,0 & 16,5 & 20,2 & 23,8 \\
\hline Febrero 2019 & 14,0 & 18,4 & 22,9 & 20,3 & 24,4 & 28,5 \\
\hline \multicolumn{7}{|l|}{ Tienen dificultades } \\
\hline Mayo 2018 & 29,8 & 33,3 & 36,8 & 34,7 & 37,8 & 41,0 \\
\hline Agosto 2018 & 26,2 & 29,1 & 32,0 & 30,1 & 33,1 & 36,1 \\
\hline Noviembre 2018 & 17,9 & 20,7 & 23,5 & 20,0 & 23,1 & 26,3 \\
\hline Febrero 2019 & 22,8 & 25,9 & 29,1 & 26,2 & 29,7 & 33,1 \\
\hline \multicolumn{7}{|c|}{ Sin grandes dificultades } \\
\hline Mayo 2018 & 43,5 & 45,9 & 48,3 & 45,8 & 48,7 & 51,5 \\
\hline Agosto 2018 & 31,8 & 34,1 & 36,3 & 38,5 & 40,9 & 43,4 \\
\hline Noviembre 2018 & 25,9 & 28,3 & 30,6 & 28,0 & 30,7 & 33,3 \\
\hline Febrero 2019 & 32,5 & 34,7 & 37,0 & 33,8 & 36,5 & 39,2 \\
\hline \multicolumn{7}{|l|}{ Pueden ahorrar } \\
\hline Mayo 2018 & 52,2 & 55,5 & 58,9 & 54,5 & 57,6 & 60,7 \\
\hline Agosto 2018 & 41,4 & 44,7 & 48,0 & 46,9 & 51,5 & 56,0 \\
\hline Noviembre 2018 & 35,7 & 39,1 & 42,6 & 40,2 & 44,2 & 48,2 \\
\hline Febrero 2019 & 44,6 & 43,3 & 52,1 & 49,0 & 45,1 & 56,0 \\
\hline
\end{tabular}

Fuente: Elaboración propia a partir de los datos de la Escuela de Estadística, Universidad de Costa Rica (2019) 


\section{ANEXO 4 \\ INTERVALOS DE CONFIANZA PARA LA DISTRIBUCIÓN PORCENTUAL DE LA CLASIFICACIÓN DE LOS CONSUMIDORES POR TIPO DE TELEFONÍA SEGÚN MES DE LA ENCUESTA}

\begin{tabular}{|c|c|c|c|c|c|c|c|}
\hline \multirow{2}{*}{$\begin{array}{l}\text { Clasificación } \\
\text { de los } \\
\text { consumidores }\end{array}$} & \multicolumn{3}{|c|}{ Tipo de encuesta } & \multicolumn{3}{|c|}{ Móvil } & \multirow{2}{*}{ Diferencia } \\
\hline & Inferior & Promedio & Superior & Inferior & Promedio & Superior & \\
\hline \multicolumn{8}{|l|}{ Pesimista } \\
\hline Mayo 2018 & 19,9 & 22,9 & 26,1 & 18,8 & 21,9 & 25,3 & 1,0 \\
\hline Agosto 2018 & 31,4 & 34,8 & 38,4 & 26,2 & 29,6 & 33,3 & 5,2 \\
\hline Noviembre 2018 & 47,8 & 51,5 & 55,2 & 45,0 & 48,9 & 52,9 & 2,6 \\
\hline Febrero 2019 & 35,6 & 39,1 & 42,8 & 33,8 & 37,6 & 41,5 & 1,5 \\
\hline \multicolumn{8}{|l|}{ Ambivalente } \\
\hline Mayo 2018 & 53,0 & 56,7 & 60,3 & 50,9 & 54,8 & 58,6 & 1,9 \\
\hline Agosto 2018 & 53,9 & 57,6 & 61,2 & 52,3 & 56,1 & 59,9 & 1,5 \\
\hline Noviembre 2018 & 37,9 & 41,5 & 45,2 & 39,0 & 42,8 & 46,7 & $-1,2$ \\
\hline Febrero 2019 & 45,6 & 49,3 & 53,0 & 45,5 & 49,4 & 53,3 & $-0,1$ \\
\hline \multicolumn{8}{|l|}{ Optimista } \\
\hline Mayo 2018 & 17,6 & 20,4 & 23,6 & 20,3 & 23,3 & 26,7 & $-2,9$ \\
\hline Agosto 2018 & 5,9 & 7,6 & 9,8 & 11,8 & 14,3 & 17,1 & $-6,7$ \\
\hline Noviembre 2018 & 5,3 & 7,0 & 9,1 & 6,4 & 8,3 & 10,7 & $-1,3$ \\
\hline Febrero 2019 & 9,4 & 11,6 & 14,2 & 10,8 & 13,0 & 15,7 & $-1,4$ \\
\hline
\end{tabular}

Nota: Para clasificar a los consumidores se utiliza la técnica de conglomerados (procedimiento de K-Medias). El objetivo es clasificarlos con base en la magnitud del ICC, agrupando consumidores que se asemejen en su confianza para lograr que entre los grupos se diferencien. La magnitud del ICC para cada grupo es fijo y en el de consumidores pesimistas es 13,6, en los ambivalentes es 43,1 y en los optimistas de 77,9.

Fuente: Elaboración propia a partir de los datos de la Escuela de Estadística, Universidad de Costa Rica (2019) 
ANEXO 5

INTERVALOS DE CONFIANZA DEL ÍNDICE DE CONFIANZA DEL CONSUMIDOR (ICC), EL ÍNDICE DE CONDICIONES ECONÓMICAS ACTUALES (ICEA) Y EL ÍNDICE DE EXPECTATIVAS ECONÓMICAS (ICEA) POR TIPO DE TELEFONÍA SEGÚN MES DE LA ENCUESTA

\begin{tabular}{|c|c|c|c|c|c|c|}
\hline \multirow{2}{*}{ Índice } & \multicolumn{3}{|c|}{ Fija } & \multicolumn{3}{|c|}{ Móvil } \\
\hline & Inferior & Promedio & Superior & Inferior & Promedio & Superior \\
\hline \multicolumn{7}{|l|}{ ICC } \\
\hline Mayo & 43,2 & 45,0 & 46,7 & 43,8 & 45,6 & 47,4 \\
\hline Agosto & 33,4 & 34,9 & 36,5 & 37,6 & 39,4 & 41,1 \\
\hline Noviembre & 26,6 & 28,2 & 29,8 & 27,9 & 29,6 & 31,4 \\
\hline Febrero & 33,1 & 34,8 & 36,5 & 34,1 & 36,0 & 37,8 \\
\hline \multicolumn{7}{|l|}{ ICEA } \\
\hline Mayo & 38,6 & 40,8 & 43,0 & 38,6 & 40,9 & 43,1 \\
\hline Agosto & 33,6 & 35,6 & 37,6 & 34,5 & 36,8 & 39,1 \\
\hline Noviembre & 25,6 & 27,6 & 29,6 & 24,2 & 26,3 & 28,4 \\
\hline Febrero & 31,2 & 33,3 & 35,4 & 31,6 & 33,9 & 36,1 \\
\hline \multicolumn{7}{|l|}{ IEE } \\
\hline Mayo & 45,7 & 47,8 & 49,9 & 46,6 & 48,8 & 51,0 \\
\hline Agosto & 32,6 & 34,5 & 36,5 & 39,1 & 41,1 & 43,2 \\
\hline Noviembre & 26,7 & 28,6 & 30,6 & 29,8 & 31,9 & 34,0 \\
\hline Febrero & 33,8 & 35,8 & 37,9 & 35,2 & 37,36 & 39,5 \\
\hline
\end{tabular}

Fuente: Elaboración propia a partir de los datos de la Escuela de Estadística, Universidad de Costa Rica (2019)

\begin{abstract}
ANEXO 6
COMPARACIÓN POR PROVINCIAS DE LAS DISTRIBUCIONES PORCENTUALES DE LAS PERSONAS DE 18 AÑOS Y MÁS DEL CENSO DE NACIONAL DE POBLACIÓN Y DE VIVIENDA 2011 Y LA ENCUESTA DE CONFIANZA DE LOS CONSUMIDORES
\end{abstract}

\begin{tabular}{lrrrrr} 
& \multicolumn{5}{c}{ Encuesta de Confianza de los Consumidores } \\
\cline { 2 - 6 } Provincia & Censo 2011 & Mayo 2018 & Agosto 2018 & Noviembre 2018 & Febrero 2019 \\
\hline Total & $\mathbf{1 0 0 , 0}$ & $\mathbf{1 0 0 , 0}$ & $\mathbf{1 0 0 , 0}$ & $\mathbf{1 0 0 , 0}$ & $\mathbf{1 0 0 , 0}$ \\
San José & 33,9 & 32,2 & 32,4 & 31,8 & 31,5 \\
Alajuela & 19,5 & 17,6 & 17,6 & 18,7 & 16,9 \\
Cartago & 11,5 & 13,7 & 11,8 & 11,8 & 10,5 \\
Heredia & 10,4 & 8,7 & 10,1 & 8,6 & 9,2 \\
Guanacaste & 7,4 & 6,9 & 7,8 & 8,0 & 8,3 \\
Puntarenas & 9,1 & 10,7 & 11,1 & 10,5 & 11,6 \\
Limón & 8,2 & 10,2 & 9,2 & 10,5 & 12,0 \\
\hline
\end{tabular}

Nota: Para el Censo se consideran las personas de 18 años y más en viviendas particulares ocupadas.

Fuente: Elaboración propia a partir de los datos de la Escuela de Estadística, Universidad de Costa Rica (2019) y a partir de Instituto Nacional de Estadística y Censos (2012). 


\begin{tabular}{|c|c|c|c|c|c|}
\hline $\begin{array}{r}\text { CO } \\
\text { PORCENTUA } \\
\text { HOGA }\end{array}$ & $\begin{array}{l}\text { ACIÓN POF } \\
\text { DE LAS PEI } \\
2018 \text { Y DE I }\end{array}$ & $\begin{array}{l}\text { REGIONES I } \\
\text { SONAS CON } \\
\text { ENCUESTA }\end{array}$ & $\begin{array}{l}\text { ANEXO } 7 \\
\text { E PLANIFICAC } \\
8 \text { AÑOS O MÁs } \\
\text { OE CONFIANZ }\end{array}$ & $\begin{array}{l}\text { ÓN DE LAS DIST } \\
\text { EN LA ENCUEST } \\
\text { DE LOS CONSUI }\end{array}$ & $\begin{array}{l}\text { BUCIONES } \\
\text { NACIONAL DE } \\
\text { DORES }\end{array}$ \\
\hline \multirow{2}{*}{$\begin{array}{l}\text { Región de } \\
\text { planificación }\end{array}$} & \multirow[b]{2}{*}{ ENAHO } & \multicolumn{4}{|c|}{ Encuesta de Confianza de los Consumidores } \\
\hline & & Mayo 2018 & Agosto 2018 & Noviembre 2018 & Febrero 2019 \\
\hline Total & 100,0 & 100,0 & 100,0 & 100,0 & 100,0 \\
\hline Central & 64,7 & 59,6 & 61,9 & 59,0 & 56,5 \\
\hline Chorotega & 7,3 & 7,0 & 8,0 & 8,3 & 8,5 \\
\hline Pacífico Central & 5,6 & 5,1 & 5,2 & 6,3 & 5,8 \\
\hline Brunca & 7,0 & 10,1 & 7,8 & 8,4 & 10,5 \\
\hline Huetar Caribe & 8,0 & 10,3 & 9,4 & 10,9 & 12,4 \\
\hline Huetar Norte & 7,4 & 7,9 & 7,6 & 7,1 & 6,2 \\
\hline
\end{tabular}

Nota: Para la ENAHO-2018 se considera las personas de 18 años o más en cuyo hogar haya al menos un celular.

Fuente: Elaboración propia a partir de los datos de la Escuela de Estadística, Universidad de Costa Rica (2019) y a partir de Instituto Nacional de Estadística y Censos (2012).

Todos los derechos reservados. Universidad de Costa Rica. Este artículo se encuentra licenciado con Creative Commons ReconocimientoNoComercial-SinObraDerivada 3.0 Costa Rica. Para mayor información escribir a revista.iice@ucr.ac.cr 